LUIZ HENRIQUE MOURÃO DO CANTO-PEREIRA

\title{
MAPEAMENTO ESPACIAL DA ATENÇÃO VISUAL ATRAVÉS DE TEMPOS DE REAÇÃO: UM ESTUDO PSICOFÍSICO
}

\author{
Tese apresentada ao Instituto de Psicologia da \\ Universidade de São Paulo como parte dos \\ Requisitos para a obtenção do grau de Doutor \\ em Neurociências e Comportamento
}

Orientador: Prof. Dr. Ronald D. Ranvaud

São Paulo 


\section{AUTORIZO A REPRODUÇÃO E DIVULGAÇÃO TOTAL OU PARCIAL DESTE TRABALHO, POR QUALQUER MEIO CONVENCIONAL OU ELETRÔNICO, PARA FINS DE ESTUDO E PESQUISA, DESDE QUE CITADA A FONTE.}

Catalogação na publicação

Serviço de Biblioteca e Documentação

Instituto de Psicologia da Universidade de São Paulo

Canto-Pereira, Luiz Henrique Mourão do.

Mapeamento espacial da atenção visual através de tempos de reação: um estudo psicofísico / Luiz Henrique Mourão do CantoPereira; orientador Ronald D. P. K. C. Ranvaud. -- São Paulo, 2006.

$92 \mathrm{p}$.

Tese (Doutorado - Programa de Pós-Graduação em Psicologia. Área de Concentração: Neurociências e Comportamento) - Instituto de Psicologia da Universidade de São Paulo.

1. Atenção 2. Distribuição espacial 3 . Tempo de reação

4. Psicofísica 5. Geoestatística I. Título.

BF321 


\section{AGRADECIMENTOS}

Eu dirijo meus agradecimentos a todos que contribuíram para a realização desse trabalho. Em particular:

- À minha família, por todo o apoio, sempre.

- Ao Prof. Ranvaud que me aceitou em seu laboratório como aluno e que me propôs a realização desse projeto de pesquisa.

- Aos colegas do laboratório de Fisiologia do Comportamento.

- Aos colegas pós-graduandos do Núcleo de Pesquisa em Neurociências e

Comportamento do Instituto de Psicologia e do Programa de Fisiologia e Biofísica do Instituto de Ciências Biomédicas.

- Aos funcionários do Instituto de Ciências Biomédicas.

- Às bibliotecárias do Instituto de Psicologia e do Instituto de Ciências Biomédicas.

- Aos voluntários que participaram dos experimentos, pela compreensão e colaboração, sem os quais não seria possível a realização deste trabalho. 
Os experimentos desta tese foram realizados no

Laboratório de Fisiologia do Comportamento do

Departamento de Fisiologia e Biofísica do Instituto de Ciências Biomédicas. 


\section{SUMÁRIO}

1 INTRODUÇÃO

2 OBJETIVO

3 MATERIAL E MÉTODOS

3.1 Sujeitos

3.2 Sala de testes

3.3 Equipamento

3.4 Procedimento

3.5 Análise geoestatística

4 RESULTADOS

4.1 Experimento I

4.2 Experimento II

4.3 Experimento III

4.4 Experimento IV

4.5 Experimento V

5 DISCUSSÃO

6 CONCLUSÃO

REFERÊNCIAS

\section{ANEXOS}

Anexo 1

Anexo 2

Anexo 3

Anexo 4

Anexo 5

Anexo 6 
Anexo 7

Anexo 8

Anexo 9 


\section{LISTA DE FIGURAS}

Figura 1. Situação experimental com o participante em posição.

Figura 2. Movimentos oculares sendo monitorados pelo experimentador.

Figura 3. Diagrama temporal de apresentação dos estímulos dos experimentos.

Figura 4A-E. Grade das posições possíveis de aparecimentos dos estímulos nos 5 experimentos. No experimento I (A) há apenas a grade espacialmente difusa que cobre toda a região da tela. Nos experimentos II, III, IV e V (B-E), além dessa grade difusa há o aparecimento de uma grade mais densa localizada no centro, esquerda, direita, e tanto na direita como na esquerda; experimentos II, III, IV e V respectivamente.

Figura 5. Ajuste do semivariograma do experimento I, o ajuste foi realizado utilizando-se o modelo esférico.

Figura 6. Mapa de pixeis do experimento I; pode-se notar o favorecimento, indicado por menores tempos de reação (cor azul) no hemicampo inferior e em especial no quadrante inferior esquerdo.

Figura 7. Ajuste do semivariograma do experimento II, o ajuste foi realizado utilizando-se o modelo Gaussiano.

Figura 8. Mapa de pixeis do experimento II; aqui pode-se perceber um favorecimento atencional da região central da tela do computador, coincidente com a região do espaço que os sujeitos experimentais deveriam atender. Note-se o aspecto alongado na horizontal do foco atencional.

Figura 9. Ajuste do semivariograma do experimento III, o ajuste foi realizado utilizando-se o modelo Gaussiano. 
Figura 10. Mapa de pixeis do experimento III, note-se o deslocamento do holofote atencional para o lado esquerdo e também uma pequena facilitação contra-lateral.

Figura 11. Ajuste do semivariograma do experimento IV, o ajuste foi realizado utilizando-se o modelo Gaussiano.

Figura 12. Mapa de pixeis do experimento IV, note-se o deslocamento do holofote atencional para o lado direito e, de maneira quase simétrica ao experimento III também uma pequena facilitação contra-lateral, mas de menor intensidade.

Figura 13. Ajuste do semivariograma do experimento V, o ajuste foi realizado utilizando-se o modelo esférico.

Figura 14. Mapa de pixeis do experimento $\mathrm{V}$, aqui pode-se verificar que há uma clara divisão da atenção, com dois focos distintos, e com menores recursos atencionais na região intermediária. 


\section{RESUMO}

CANTO-PEREIRA, L. H. M. Mapeamento espacial da atenção visual através de

tempos de reação: Um estudo psicofísico. São Paulo, 2006. 92 p. Tese (Doutorado).

Núcleo de Pesquisa em Neurociências e Comportamento, Instituto de Psicologia,

Universidade de São Paulo.

Nos últimos vinte e cinco anos a atenção visual tem sido descrita em termos de diversas metáforas, salientando-se o holfote atencional, a lente zoom e o gradiente atencional. Um aspecto essencial dessas metáforas é a distribuição dos recursos atencionais no campo visual, que não é necessariamente determinado pelo ponto de fixação e que pode depender tanto de eventos externos como também de estados mentais. Esse estudo apresenta uma abordagem inovadora, baseada em tempos de reação analisados através de métodos geoestatísticos, para investigar como a atenção visual se distribui em áreas extensas do campo visual. Cinco diferentes experimentos foram realizados: no experimento I participantes foram solicitados a não atender nenhuma região em particular do espaço (da tela do computador) caracterizando assim a atenção difusa. Nos demais experimentos, os participantes foram solicitados a direcionar a atenção explicitamente ao centro (expt. II); de maneira encoberta para esquerda (expt. III) ou para direita (expt. IV); ou dividindo a atenção em duas regiões tanto a direita como a esquerda simultaneamente, mas não para o centro (expt. V). A distribuição espacial da atenção, medida através de tempos de reação, foi obtida na forma de mapa de pixieis resultante da análise geoestatísica. O experimento I teve como resultado um favorecimento atencional do quadrante nasal do hemicampo inferior. O experimento II, como esperado, apresentou um foco atencional coincidente com o ponto de fixação. Os experimentos III e IV mostraram claramente focos atencionais para esquerda e direita, respectivamente. Por fim o experimento V mostrou dois focos atencionais, à esquerda e direita, indicando divisão atencional. Esses resultados demonstram a utilidade da geoestatística para a análise de tempos de reação no estudo da atenção visual. O método também forneceu evidências claras da possibilidade de dividir a atenção visual à direita e à esquerda do ponto de fixação, um tema ainda controverso na literatura. 


\begin{abstract}
CANTO-PEREIRA, L. H. M. Mapping the spatial distribution of visual attention

through reaction times: A psychophysical study. São Paulo, 2006. 92 p. Thesis.

Núcleo de Pesquisa em Neurociências e Comportamento, Instituto de Psicologia, Universidade de São Paulo.
\end{abstract}

In the past twenty five years visual attention has been described in terms of varied metaphors, among which a spotlight, a zoom lens and a gradient field. An essential aspect of all these metaphors is the distribution of attentional resources in the visual field, which is not necessarily determined by the fixation point, and may depend on both external events and internal mental processes. This study presents a novel approach, based on reaction times and analyzing data with geostatistical techniques, to investigate how visual attention is allocated in large continuous regions of space. Five different experiments were performed: in experiment I participants were asked not to attend to any particular region, but rather try to spread their attention as uniformly as possible over the computer screen (diffuse attention). In the remaining experiments, participants were instructed to direct their visual attention overtly towards the center (expt. II), or covertly to the left (expt. III) to the right (expt. IV), or to divide their attention attending regions both right and left (but not to the center (expt. V). The spatial distribution of attention, as evaluated through reaction times, was obtained in the form of pixel maps resulting from the geostatistical analysis. Experiment I showed a lower hemifield advantage, in the nasal quadrant. Experiment II, as expected, presented an atentional focus coincident with the fixation point. Experiments IV and V showed clear attentional foci, to the left and to the right, respectively. Finally, Experiment V showed two clear lateral foci, one to the left, the other to the right, indicating attentional division. These results demonstrate the usefulness of geostatistics to analyze reaction time data for the study of visual attention. The method also provided clear evidence for the ability of subjects to divide their visual attention in two well separated foci, to the right and to the left of their fixation point, an issue still debated in the literature. 


\section{INTRODUÇÃO}

Nos últimos vinte anos a atenção visual tem sido descrita através de várias metáforas e / ou modelos que tentam descrever aspectos essenciais desse fenômeno; desses, os que apresentaram maior repercussão são os seguintes:

- "holofote atencional"; esse modelo (POSNER e PETERSEN, 1990) engloba duas das principais características da atenção visual, (i) a atenção seria espacialmente delimitada e (ii) existiria um custo temporal para seu deslocamento; de acordo com esse modelo o foco atencional dificilmente poderia ser dividido, da mesma maneira que um holofote de luz ilumina apenas uma região do espaço em um dado momento;

- "lente zoom"; nesse modelo, proposto por Eriksen e St. James (1986), o tamanho do foco atencional seria variável, podendo se adaptar à tarefa a ser executada; apresentaria uma distribuição uniforme de recursos atencionais na área atendida, de forma que quanto maior a área atendida menor a densidade de tais recursos; e

- "gradiente atencional"; tanto o tamanho como a forma e densidade do foco atencional seriam variáveis, estando mais concentrados no seu centro e diminuídos na sua periferia (DOWNING e PINKER, 1985; SHULMAN et al., 1986).

Outros modelos, mais recentes, e com alguma repercussão, são:

- "modelo modificado de atenção visuo-espacial" de Kraft et al. (2005) que apresenta como principal característica permitir divisão da atenção sendo cada hemisfério capaz do controle simultâneo de um foco atencional; e 
- “chapéu mexicano" (MÜLLER et al., 2005), pelo qual ao redor do foco atencional haveria primeiro um anel de menor densidade, seguido mais perifericamente de um segundo anel com densidade um pouco maior.

Um aspecto essencial de todas essas metáforas é a distribuição espacial dos recursos atencionais no campo visual. Esses recursos não são determinados necessariamente pelo foveamento, ou seja, independem do ponto de fixação visual.

Uma questão que tem sido extensivamente debatida diz respeito à possibilidade da distribuição da atenção no espaço poder ser dividida em locais não contíguos; em um recente trabalho Awh e Pashler (2000) apresentaram evidencias em favor da possibilidade de divisão do foco atencional, contrariando diversos trabalhos anteriores (ERIKSEN e YEH, 1985; CASTIELLO e UMILTA, 1990; PAN e ERIKSEN, 1993; HEINZE et al., 1994; KRAMER e HAHN, 1995; EIMER, 1999).

Recentemente técnicas de imageamento, como Ressonância Magnética Funcional (fMRI) e Potenciais Relacionados a Eventos (ERP) têm sido introduzidas no estudo da distribuição espacial da atenção. Por meio da primeira dessas técnicas (fMRI), Brefzynski e DeYoe (1999) propuseram um correlato fisiológico da metáfora do "holofote atencional". Müller et al. (2003), usando técnicas eletrofisiológicas (ERP) demonstraram ser possível a divisão do foco atencional; Gobell et al. (2004) também sugerem que haveria a possibilidade de dividir a atenção.

Uma questão interessante é a medida da alocação dos recursos atencionais, ou seja, a medida, ponto a ponto, da densidade do "holofote atencional", que essas técnicas começam a abordar. Para verificar a coerência dessas medidas com técnicas mais tradicionais (psicofísicas) de medida, torna-se necessária uma investigação extensa do campo visual, cobrindo uma região maior que apenas poucos pontos, como em todos os protocolos empregados até o momento. Uma limitação dos experimentos 
relatados na literatura até o presente é a descontinuidade espacial da amostragem. Para caracterizar a distribuição espacial da atenção visual torna-se necessário amostrar o campo visual com uma grade de estímulos de densidade suficiente para verificar os padrões espaciais do campo atencional com a resolução desejada.

Nos experimentos clássicos a distribuição espacial da atenção visual foi avaliada pela rapidez de respostas motoras (medidos os tempos de reação) a estímulos visuais apresentados em apenas alguns poucos pontos, mas com um grande número de repetições. Isso se deve, sobretudo, a variabilidade dos tempos de reação, que é grande em comparação com o tamanho do efeito a ser medido, que induz à coleta de um grande número de respostas para reduzir o erro padrão. Devido a isso o campo visual investigado sempre ficou restrito a apenas alguns poucos graus de ângulo visual. Sendo possível determinar como se distribuiria a atenção visual no espaço, surgem múltiplas questões:

- o holofote atencional focado no ponto foveado difere do holofote direcionado lateralmente, de forma encoberta descrita como a habilidade de selecionar uma dada região do campo visual sem movimentos oculares e / ou da cabeça naquela direção, mas dando prioridade no processamento perceptual dessa região (BALDASSI e BURR, 2000; CARRRASCO e MCELREE, 2001; CARRASCO et al., 2001; CARRASCO et al., 2002)?

- como será distribuída a atenção, se houver divisão do holofote em duas regiões não contíguas do espaço?

Nossa proposta apresenta uma mudança significativa dos protocolos experimentais tradicionalmente empregados na investigação psicofísica da atenção, ou seja, ao contrário de amostrar poucos pontos diversas vezes, propomos amostrar muitos pontos poucas vezes (ou eventualmente uma única vez), sendo possível assim 
amostrar de forma contínua uma área muito maior do campo visual, com o mesmo esforço experimental. Para a análise dos dados, adotamos um método (geoestatístico) diferente dos clássicos (ANOVA e derivados, Modelo Linear Geral, teste t, etc), focando a dependência espacial dos dados obtidos.

Krige e outros, na década de 50, motivados por problemas ligados à mineração de jazidas de ouro e outros metais preciosos, deram início ao desenvolvimento de uma ferramenta de análise estatística baseada na dependência espacial de dados. Essa metodologia, denominada geoestatística, analisa inicialmente a variância de diferenças na variável de interesse (no nosso caso tempos de reação) em função da distância entre os dados amostrais, e usa tal informação para descrever a dependência espacial do fenômeno estudado (JOURNEL, 1986). Esse método é extensamente utilizado em geologia, hidrologia, oceanografia e agricultura (GOOVAERTS, 1997; YAMAMOTO, 2000) e mais recentemente tem sido empregado em estudos epidemiológicos e em outros fenômenos biológicos (FUCHS e DEUTZ, 2002; MATHER et al., 2004; LUDOVISIA et al., 2005; MIRANDA e DOLLINOY, 2005).

Assim, o presente trabalho investigou a distribuição espacial da atenção visual por técnica psicofísica, medindo tempos de reação simples a estímulos apresentados em uma área extensa $\left(24^{\circ}\right.$ por $\left.16^{\circ}\right)$ e contínua do campo visual, em diferentes condições experimentais, de forma a investigar questões clássicas de atenção encoberta e dividida, aplicando métodos geoestatísticos para a análise dos dados. 


\section{OBJETIVO}

O presente trabalho tem por objetivo:

(i) demonstrar a viabilidade de mapear a distribuição espacial da atenção visual através da medida de tempos de reação simples a estímulos apresentados em regiões amplas do campo visual analisando os dados por métodos geoestatísticos;

(ii) aplicar essa técnica inovadora ao mapeamento da atenção visual em diferentes condições experimentais, para comparar as situações de atenção difusa, atenção explícita, atenção encoberta e atenção dividida. 


\section{MATERIAIS E MÉTODOS}

\subsection{Sujeitos}

Foram utilizados 30 sujeitos experimentais na faixa etária de 18 a 30 anos, igualmente distribuídos entre os sexos, alunos de graduação e pós-graduação de disciplinas em curso no Instituto de Ciências Biomédicas da Universidade de São Paulo. Os sujeitos foram contatados na sala de aula, mediante prévia autorização do docente responsável, e aqueles que desejaram participar como voluntários se inscreveram em uma lista e foram contatados posteriormente para participarem dos testes psicofísicos.

Após o preenchimento do Termo de Consentimento Livre e Esclarecido (anexo 1) os sujeitos experimentais foram submetidos ao seguinte protocolo:

- avaliação clínica composta de: anamnese (anexo 2) e teste de acuidade visual Snellen medida pelo teste computadorizado "Freiburg Visual Acuity \& Contrast Test" (BACH, 1997).

- teste de dominância ocular; todos os experimentos foram realizados na situação monocular (olho dominante), o olho não dominante foi ocluído com um tampão elástico.

Foram adotados os seguintes critérios de inclusão / exclusão:

- Critérios de inclusão: acuidade visual Snellen 20/25, ou melhor, com correção; dominância manual direita.

- Critérios de exclusão: presença de patologias sistêmicas e / ou oftalmológicas que possam comprometer os resultados tais como diabetes mellitus, 
hipertensão arterial, glaucoma; uso crônico de medicamentos, consumo regular de álcool, fumo ou outras drogas.

\subsection{Sala de testes}

As sessões experimentais foram realizadas em uma sala de testes escura com isolamento acústico relativo do Laboratório de Fisiologia do Comportamento do Instituto de Ciências Biomédicas da Universidade de São Paulo. Os sujeitos experimentais foram posicionados a $57 \mathrm{~cm}$ do monitor. A essa distância $1 \mathrm{~cm}$ na tela do monitor corresponde a $1^{\circ}$ de ângulo visual (Figura 1).

\subsection{Equipamento}

O equipamento utilizado foi um microcomputador Athlon XP 2400+ 2.0 GHz, sistema operacional Windows 2000, placa de som Encore Crystal SoundFusion CS4281, placa de vídeo de 10 bits Matrox Millennium P-650 dual head, monitor de vídeo de 19' Samsung modelo Syncmaster 997 DF, com uma taxa de renovação (refresh rate) de $100 \mathrm{~Hz}$ e resolução de 800x600. Para aquisição das respostas foi utilizado um gamepad Leadership de 2 botões modelo JPD310 conectado ao microcomputador pela porta gameport. A utilização do gamepad através da porta gameport garantia precisão e acurácia da ordem de 1 milisegundo (SEGALOWITZ e GRAVES, 1990).

A sessão experimental foi sempre acompanhada pelo experimentador, em tempo real, através de um segundo monitor de vídeo de 17' configurado para exibir a mesma tela que o monitor localizado na sala de testes. O experimentador monitorou a fixação do olhar do sujeito experimental durante os experimentos através de um 
monitor de vídeo de 20’ conectado a uma micro-câmera de vídeo sensível ao infravermelho modelo VT-686CCD, posicionada de tal maneira a permitir detecções de movimentos oculares da ordem de $1^{\circ}$ (Figura 2).

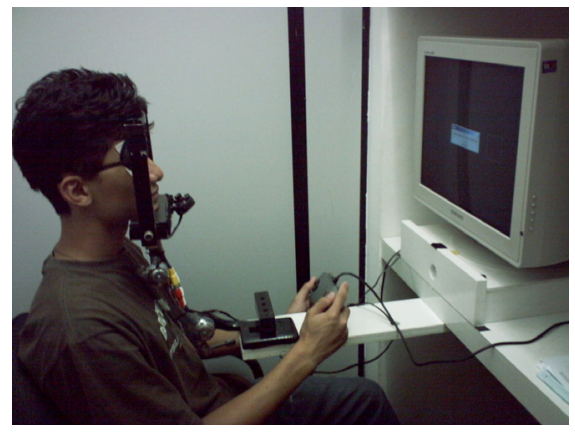

Figura 1: Situação experimental com o participante em posição.



Figura 2: Experimentador acompanhando a sessão experimental e monitorando os movimentos oculares.

Os experimentos foram realizados através do programa de computador EPrime (E-Prime v. 1.1.4 - Psychology Software Tools Inc.) (SCHNEIDER et al., 2002a; SCHNEIDER et al., 2002b) que gerenciou tanto a apresentação dos estímulos visuais como a coleta das respostas dos sujeitos experimentais. 


\subsection{Procedimento}

Tomando como premissa que tempos de reação a estímulos visuais são uma medida confiável e tradicionalmente utilizada para quantificar atenção visual (POSNER, 1980; POSNER et al., 1980) e que a atenção visual apresenta dependência espacial, apresentamos estímulos visuais dispostos em uma grade regular com 82, 154 ou 158 pontos amostrais (dependendo do experimento), sempre abrangendo de forma contínua um ângulo visual de $24^{\circ}$ por $16^{\circ}$ (nas direções horizontal e vertical, respectivamente).

O protocolo experimental adotado constou de uma série de cinco experimentos em que os participantes teriam que fixar, sempre, o olhar em uma cruz no centro da tela do computador, e apertar um botão do gamepad com o dedo indicador da mão dominante (direita) assim que aparecesse um estímulo visual; os experimentos empregados consistiram em:

- experimento I (estudo de atenção difusa): estímulos distribuídos uniformemente em toda a tela do computador;

- experimento II (estudo de atenção explícita): maior densidade de estímulos no centro da tela do computador, ou seja, nas imediações do ponto de fixação;

- experimentos III e IV (estudo de atenção encoberta): maior densidade de estímulos em regiões periféricas laterais da tela do computador; e

- experimento V (estudo de atenção dividida): maior densidade de estímulos em ambas as regiões periféricas laterais da tela do computador.

A Figura 3 mostra o diagrama temporal de apresentação dos estímulos nos cinco experimentos. 


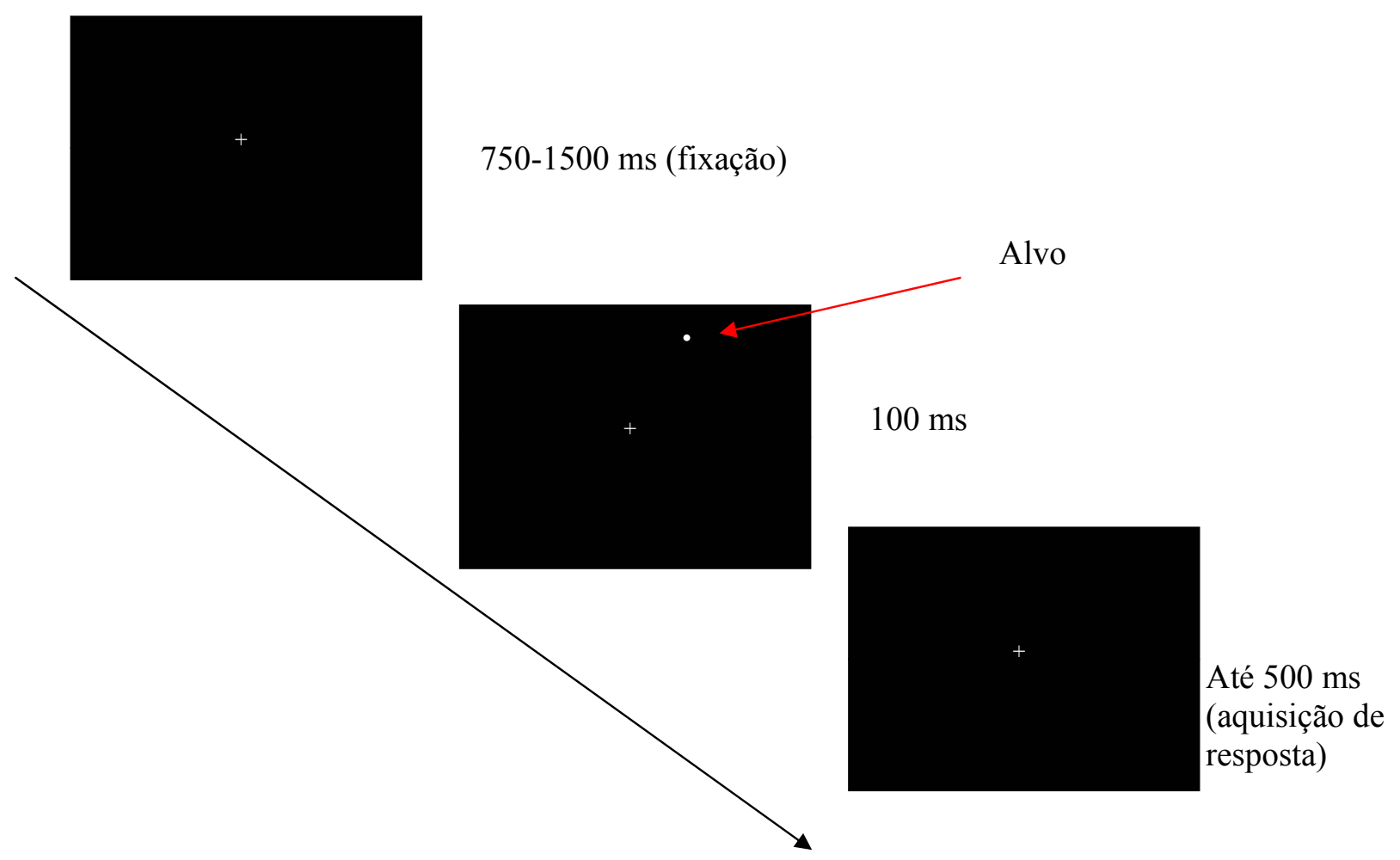

Figura 3: Diagrama temporal de apresentação dos estímulos dos experimentos.

Antes da sessão experimental foi realizada uma sessão de treinamento, que teve por objetivo familiarizar os sujeitos experimentais com a situação dos testes e a aquisição de respostas através do gamepad.

Todos os experimentos consistiram em medir o tempo de reação simples frente à apresentação de um alvo com um ângulo visual de $0,2^{\circ}$, luminância de $80 \mathrm{~cd} / \mathrm{m}^{2}$, contra um fundo preto $\left(0 \mathrm{~cd} / \mathrm{m}^{2}\right)$, apresentado em diferentes posições na tela do computador; os alvos tiveram duração de 100 ms, essa breve duração foi utilizada para evitar tanto a ocorrência de movimentos oculares como a possibilidade, levantada por MÜLLER et al. 2003, de desvios atencionais entre diferentes regiões do espaço. 
Foram consideradas respostas válidas as compreendidas entre 150 e $500 \mathrm{~ms}$ a partir da apresentação do alvo; respostas emitidas abaixo de $150 \mathrm{~ms}$ foram consideradas antecipações e as acima de 500 ms não-respostas. Também tentativas com perdas de fixação do olhar foram invalidadas.

As Figuras 4A-E mostram as posições em que os alvos foram apresentados nos cinco experimentos. A grade de alvos equivale a um ângulo visual de $24^{\circ} \times 16^{\circ}$ e cada ponto da grade foi apresentado apenas uma vez por experimento. O intervalo entre alvos era aleatorizado entre 750 e $1500 \mathrm{~ms}$. Em todos os experimentos os sujeitos eram instruídos a prestar atenção dentro da(s) molduras cinza(s), se presentes.

No experimento I (Figura 4A) os estímulos eram apresentados em uma grade de 82 tentativas (trials) de mesma densidade, ou seja, igualmente distribuídos na tela do monitor, esse experimento funcionou como linha de base dos tempos de reação em função da excentricidade dos estímulos, não havendo, portanto, um foco atencional preferencial, estando a atenção difusamente distribuída.

No experimento II (Figura 4B) os estímulos eram apresentados em uma grade com 158 tentativas com uma maior densidade no centro da tela, a região central que compreende um ângulo visual de $4^{\circ} \times 4^{\circ}$ foi delimitada por uma moldura cinza com uma luminância de $20 \mathrm{~cd} / \mathrm{m}^{2}$, apresentando a mesma probabilidade de aparecimento de estímulos que o restante do monitor, favorecendo o direcionamento do foco atencional para o centro da tela. 

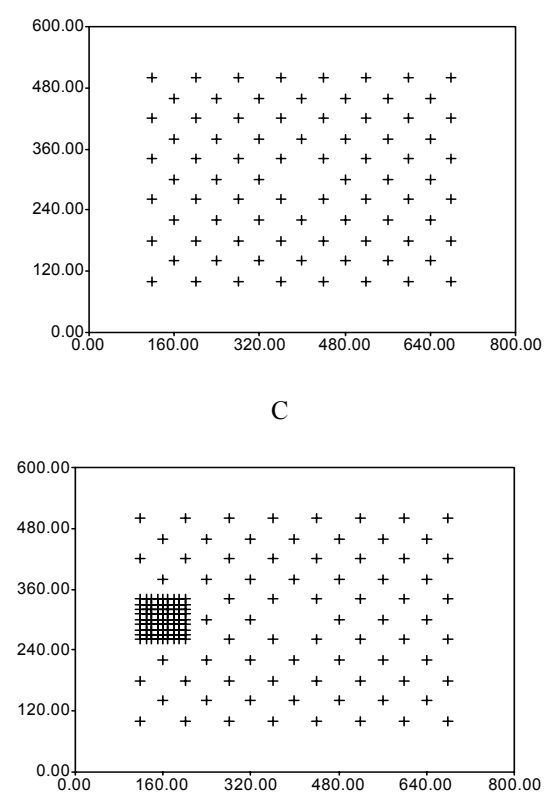

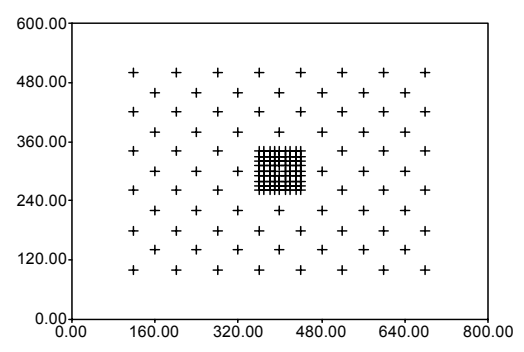

D

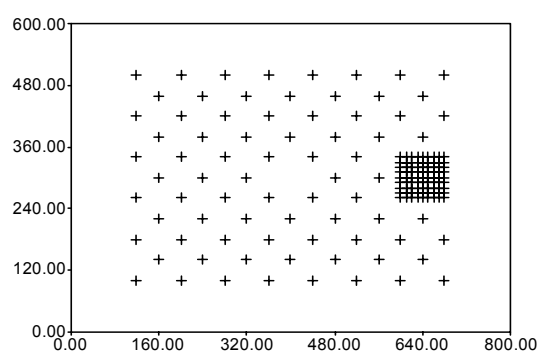

E

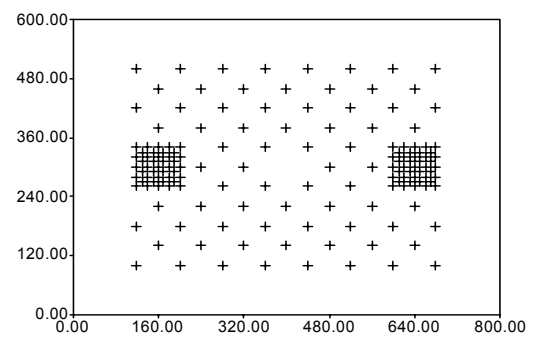

Figura 4. A-E: Grade das posições possíveis de aparecimentos dos estímulos nos cinco experimentos. No experimento I (A) há apenas a grade espacialmente difusa que cobre toda a região da tela. Nos experimentos II, III, IV e V (B-E), além dessa grade difusa há o aparecimento de uma grade mais densa (delimitada por uma moldura cinza) localizada no centro, esquerda, direita, e tanto na direita como na esquerda (experimentos II, III, IV e V respectivamente). Os eixos x e y estão expressos em pixeis (resolução do monitor)

Nos experimentos III e IV (Figuras 4C e 4D) os estímulos eram apresentados em uma grade de 158 tentativas com uma maior densidade no lado esquerdo e direito da tela, respectivamente, ou seja, nesses experimentos a área do monitor que se encontra a $10^{\circ}$ de excentricidade perfazendo uma região de ângulo visual de $4^{\circ} \times 4^{\circ}$ 
também delimitada por uma moldura cinza com uma luminância de $20 \mathrm{~cd} / \mathrm{m}^{2}$, apresentando a mesma probabilidade de aparecimento de estímulos que o restante do monitor. Dessa forma o foco atencional foi direcionado para um dos lados da tela.

No experimento V (Figura 4E) os estímulos eram apresentados em uma grade de 154 pontos com uma maior densidade nos dois lados, direito e esquerdo, nesse experimento as áreas do monitor que se encontram a $10^{\circ}$ de excentricidade em uma região de ângulo visual de $4^{\circ} \times 4^{\circ}$ também delimitada por duas molduras cinza com uma luminância de $20 \mathrm{~cd} / \mathrm{m}^{2}$; havia a mesma probabilidade de aparecimento de estímulos que o restante do monitor, fazendo com que o foco atencional esteja dividido, estando direcionado tanto para o lado direito como para o lado esquerdo da tela.

\subsection{Análise geoestatística}

A geoestatística se baseia em diferenças da variável de interesse em função de sua localização espacial; se pares de dados forem espacialmente independentes, mostrariam a mesma variabilidade, independentemente da localização dos pontos em que foram colhidos, e esse método não poderia ser empregado (JOURNEL, 1986; GOOVAERTS, 1997). Dados colhidos em pontos próximos entre, si mostram menor variabilidade do que dados que se encontram separados por distâncias maiores, se apresentarem dependência espacial. A natureza desse padrão de variabilidade é característica de um conjunto de dados amostrais. Essa variabilidade é computada em uma função chamada semivariograma.

O método geoestatístico engloba as seguintes etapas:

- computação do semivariograma (Equação i), para verificar a dependência espacial da variável de interesse (no caso, tempos de reação); 
- ajuste (fitting) do semivariograma com um, dentre vários, modelos (no nosso caso, funções esférica, Gaussiana ou exponencial);

- obtenção dos mapas de pixeis, através de um método de interpolação, próprio da geoestatística, conhecido como krigagem ordinária.

(Equação i) $\quad \gamma(h)=\frac{1}{2 n} \cdot \sum_{i=1}^{n}[Z(x+h)-Z(x)]^{2}$,

onde, $\gamma(h)$ é o semivariograma em função de h, que é a distância entre pares de pontos amostrados; $\mathrm{n}$ é o número de pares de pontos separados por uma distância $\mathrm{h}$; $\mathrm{Z}(\mathrm{x})$ é o valor da variável (nosso caso, tempo de reação) regionalizada no ponto x; $\mathrm{Z}(\mathrm{x}+\mathrm{h})$ é o valor da variável regionalizada no ponto $(\mathrm{x}+\mathrm{h})$.

Semivariogramas são computados para medir as relações espaciais dos tempos de reação e ajustados em modelos teóricos segundo funções esférica (Equação ii), Gaussiana (Equação iii) ou exponencial (Equação iv):

(Equação ii)

$$
\begin{aligned}
& \gamma(h)=C_{0}+C\left[\frac{3}{2}\left(\frac{h}{a}\right)-\frac{1}{2}\left(\frac{h}{a}\right)^{3}\right],(\text { para } \mathrm{h}<\mathrm{a}), \mathrm{e} \\
& \gamma(h)=C_{0}+C,(\text { para } \mathrm{h} \geq \mathrm{a}) ;
\end{aligned}
$$

(Equação iii)

$$
\gamma(h)=C_{0}+C\left[1-\exp \left(-\left(\frac{h}{a}\right)^{2}\right)\right]
$$


(Equação iv) $\quad \gamma(h)=C_{0}+C\left[1-\exp \left(-\left(\frac{h}{a}\right)\right)\right]$

onde, $\mathrm{C}$ e $\mathrm{C}_{0}$ são parâmetros de variância (respectivamente total e aleatória, e a diferença entre esses dois valores é conhecida como variância espacial), e $a$ é a amplitude, ou seja a distância além da qual não há mais dependência espacial.

Para se obter mapas de pixeis, um método de interpolação é empregado; em geoestatística o método adotado é conhecido como krigagem (Equação v). Esse método de interpolação estima valores em pontos não amostrados a partir de dados obtidos em locais amostrados; o semivariograma determina a variância espacial, e é utilizado para minimizar o erro do valor estimado. Krigagem é um conjunto de algoritmos com o objetivo de obter a melhor estimativa possível em locais não amostrados de maneira a minimizar o erro nesse local. A estimativa de krigagem consiste na resolução de uma matriz de equações lineares (JOURNEL, 1986; GOOVAERTS, 1997).

$$
\text { (Equação v) } Z^{*}\left(x_{0}\right)=\sum_{i=1}^{n} \lambda_{1} \cdot Z\left(x_{i}\right)
$$

onde, $Z^{*}\left(\mathrm{x}_{0}\right)$ é a estimativa de krigagem, e os fatores ponderados $\left(\lambda_{1}, \mathrm{i}=1, \mathrm{n}\right)$ são o resultado da solução de um sistema de equações lineares.

Os dados dos experimentos foram submetidos à análise geoestatística pelo programa de computador GeoVisual (YAMAMOTO, 2002). 


\section{RESULTADOS}

Em um primeiro trabalho, foi possível demonstrar a viabilidade do método descrito para a investigação de interesse, ou seja, para a determinação da distribuição espacial da atenção (anexo 3). Esses resultados iniciais não demonstraram claramente a possibilidade de desengajamento da atenção do ponto de fixação. Nesse trabalho inicial foi utilizado como fundo da tela do computador luminância média $\left(20 \mathrm{~cd} / \mathrm{m}^{2}\right)$, além disso, não havia a presença de molduras indicando claramente para onde os sujeitos deveriam orientar a atenção visual.

Isso nos motivou a fazer algumas mudanças no protocolo experimental (detalhadas na seção Materiais e Métodos), a saber:

(i) a inclusão de uma moldura na região que os voluntários deveriam atender, "ancorando" a atenção naquela região do monitor, e

(ii) mudança do fundo para preto $\left(0 \mathrm{~cd} / \mathrm{m}^{2}\right)$, no lugar de luminância média (20 $\mathrm{cd} / \mathrm{m}^{2}$ ), para aumentar o contraste do alvo facilitando a tarefa de detecção, importante sobretudo em excentricidades maiores.

Os resultados obtidos na série definitiva de cinco experimentos estão apresentados abaixo. Esses resultados são os dados médios dos sujeitos experimentais, cada um contribuindo com apenas um tempo de reação para cada posição amostrada no campo visual, e com dominância ocular direita. Optamos por apresentar graficamente os resultados da seguinte forma: (i) o semivariograma de cada experimento, com o ajuste (fitting) realizado, mostrando a presença de dependência espacial em todos os experimentos realizados, o que autoriza o uso de análise geoestatística; e (ii) o mapa de pixeis, obtido através do método de krigagem ordinária. Os mapas de pixeis são codificados por cores indo do azul (tempos de 
reação mais baixos, indicando alta concentração de atenção), ao vermelho (longos tempos de reação, indicando baixa densidade atencional).

\subsection{Experimento I}

Experimento de atenção difusa; nesse experimento o ajuste do semivariograma (Figura 5) foi realizado utilizando-se o modelo esférico; pode-se notar nesse ajuste a existência de um grande componente aleatório (intersecção da curva ajustada na ordenada aproximadamente em valor alto, de 115; ou seja esse componente aleatório conhecido como efeito pepita - é responsável por quase $60 \%$ do ajuste, $i$. e. da variância total, da ordem de 205, o efeito pepita contribui com 115). Pode-se notar no mapa de pixeis do experimento I (Figura 6) que houve um privilégio atencional do hemicampo inferior e em especial do quadrante inferior esquerdo (nasal), representado por menores tempos de reação.

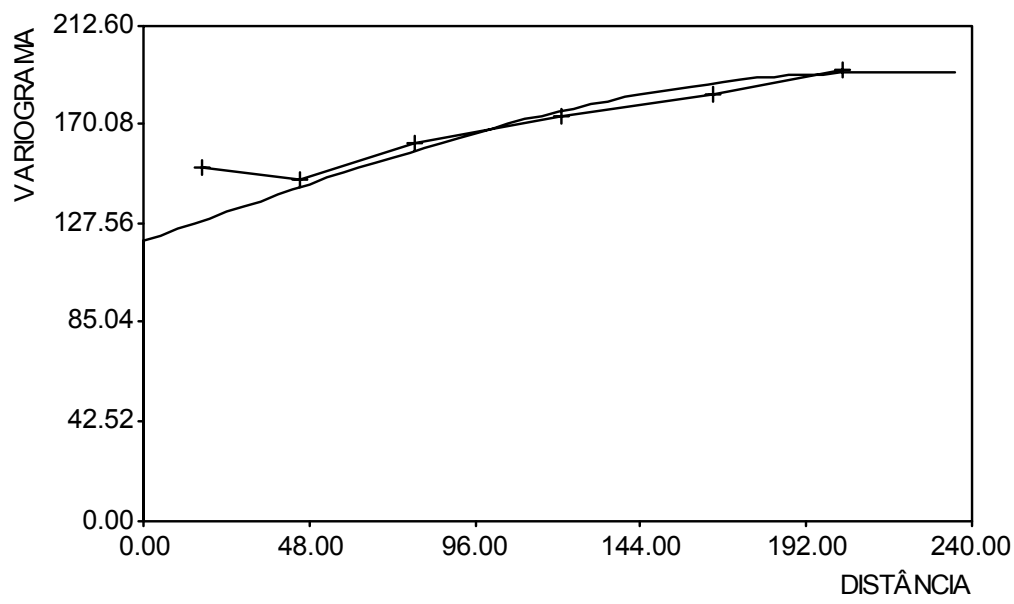

Figura 5: Ajuste do semivariograma do experimento I, o ajuste foi realizado utilizando-se o modelo esférico. 


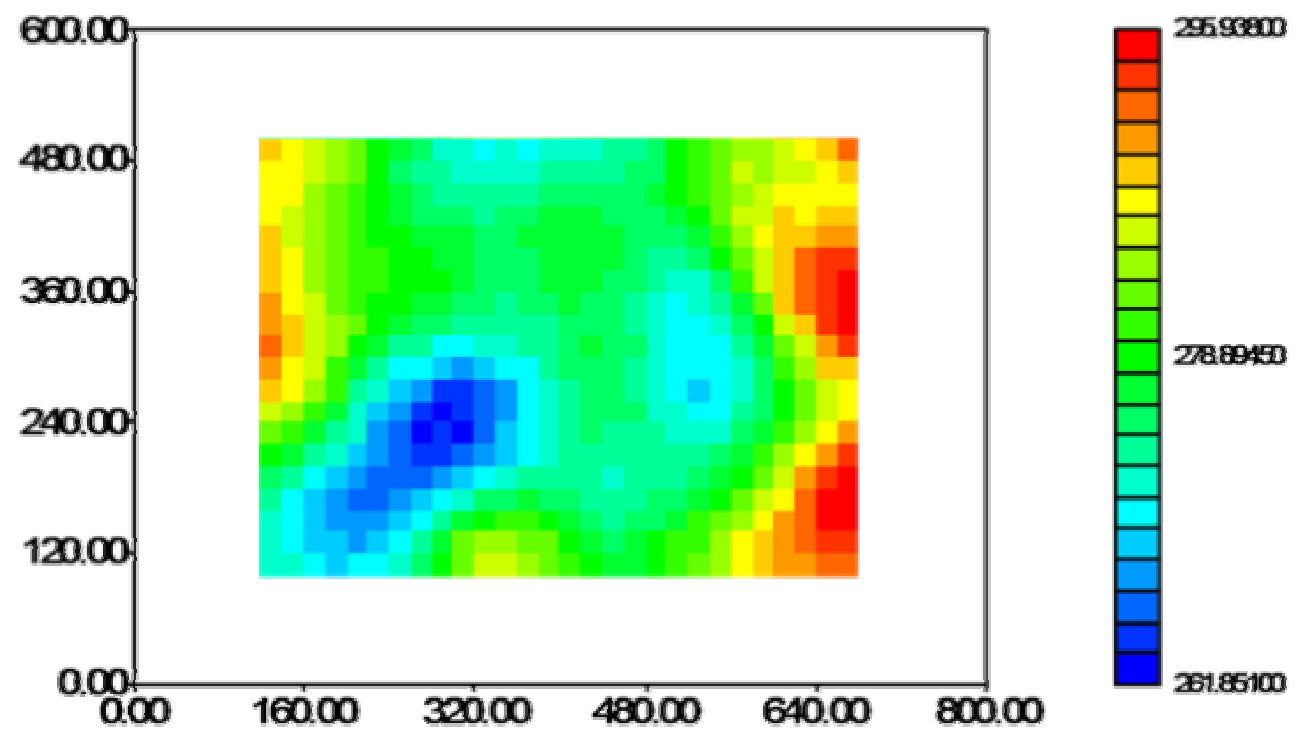

Figura 6: Mapa de pixeis do experimento I; pode-se notar o favorecimento, indicado por menores tempos de reação (cor azul) no hemicampo inferior e em especial no quadrante inferior esquerdo.

\subsection{Experimento II}

Experimento de atenção explícita; nesse experimento o ajuste do semivariograma (Figura 7) foi realizado utilizando-se o modelo Gaussiano, que apresenta maior continuidade espacial. Podemos notar no mapa de pixeis do experimento II (Figura 8) que houve de fato um privilégio atencional da região central da tela, coincidente com o ponto de fixação, região que os sujeitos experimentais deveriam atender. 


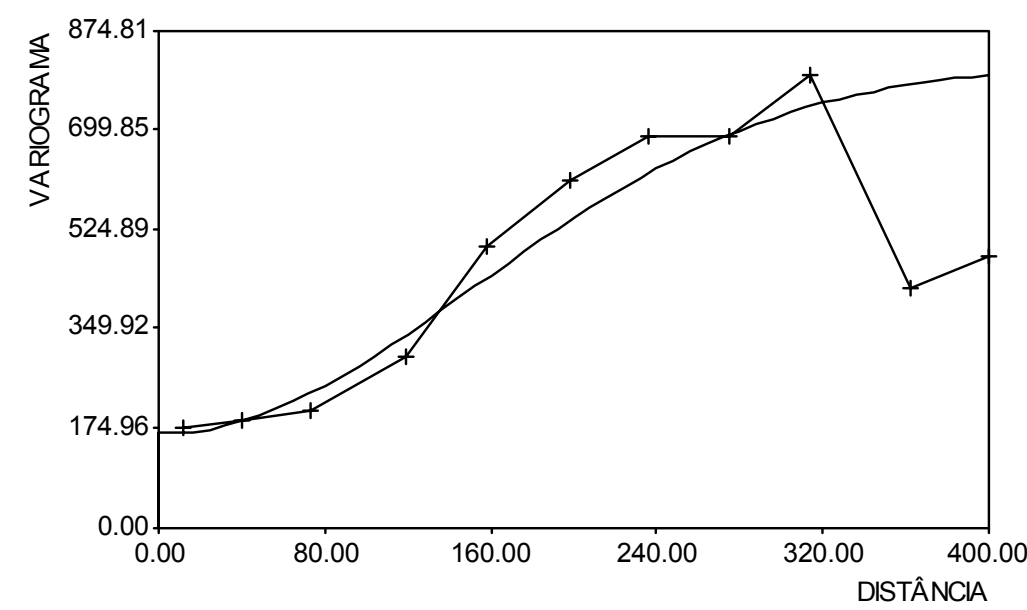

Figura 7: Ajuste do semivariograma do experimento II, o ajuste foi realizado utilizando-se o modelo Gaussiano.
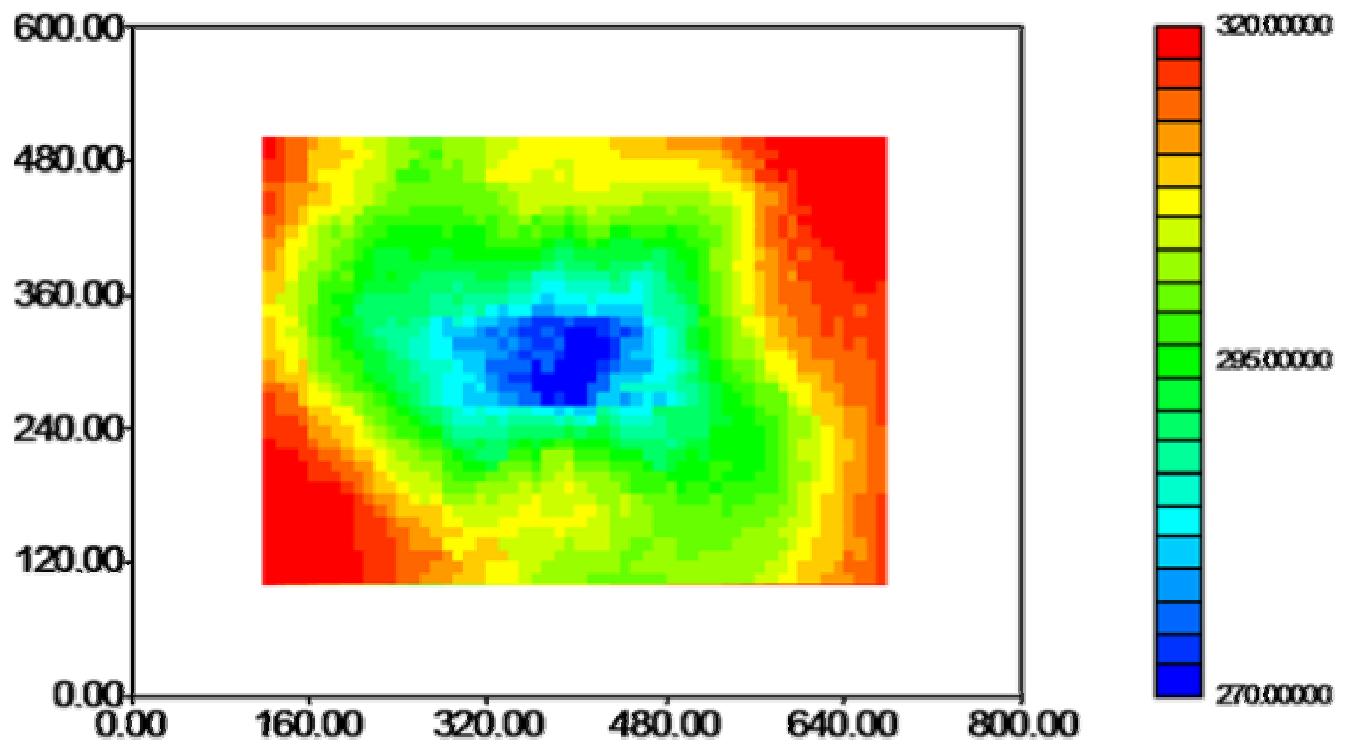

Figura 8: Mapa de pixeis do experimento II; aqui pode-se perceber um favorecimento atencional da região central da tela do computador, coincidente com a região do espaço que os sujeitos experimentais deveriam atender. Note-se o aspecto alongado na horizontal do foco atencional. 


\subsection{Experimento III}

Experimento de atenção encoberta (foco atencional do lado esquerdo); nesse experimento o ajuste do semivariograma (Figura 9) foi realizado utilizando-se o modelo Gaussiano. Pode-se notar no mapa de pixeis desse experimento (Figura 10) que houve um privilégio atencional da região que os sujeitos experimentais deveriam atender, ou seja, do lado esquerdo; interessante notar que também houve uma pequena facilitação contra-lateral.

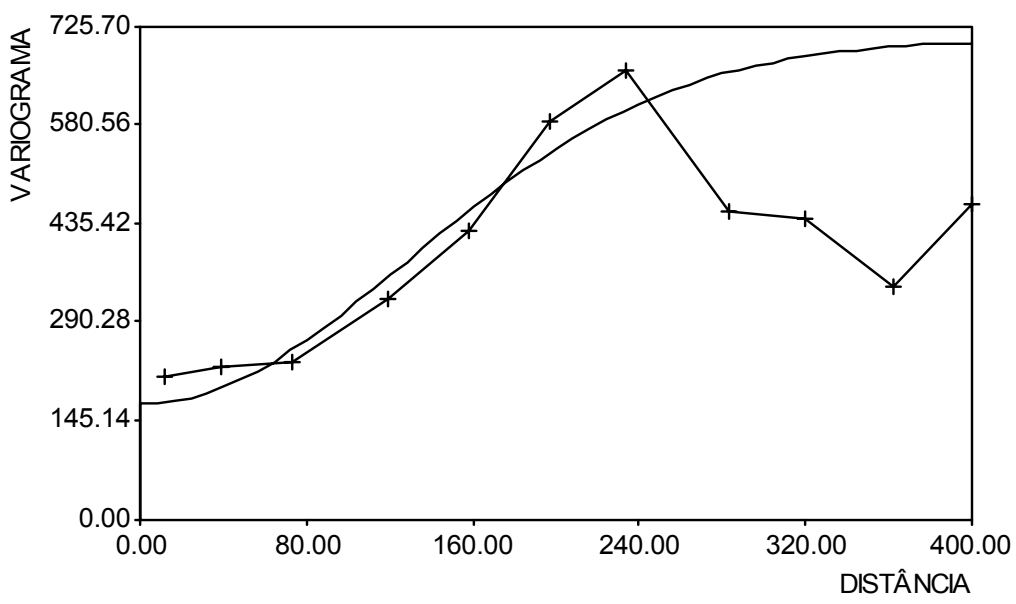

Figura 9: Ajuste do semivariograma do experimento III, o ajuste foi realizado utilizando-se o modelo Gaussiano. 


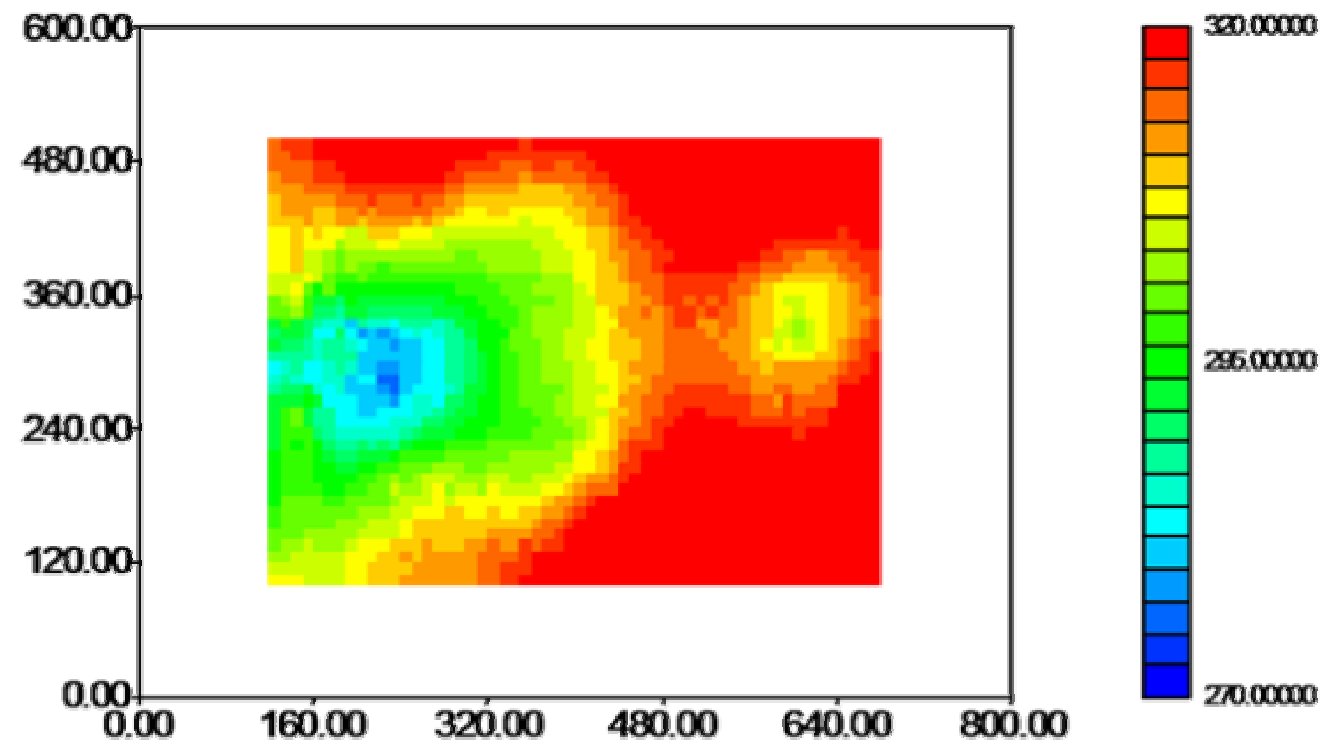

Figura 10: Mapa de pixeis do experimento III, note-se o deslocamento do holofote atencional para o lado esquerdo e também uma pequena facilitação contra-lateral.

\subsection{Experimento IV}

Experimento de atenção encoberta, simétrico ao anterior (foco atencional do lado direito); nesse experimento o ajuste do semivariograma (Figura 11) foi realizado utilizando-se o modelo Gaussiano. Pode-se notar no mapa de pixeis desse experimento (Figura 12) que houve um privilégio atencional da região que os sujeitos experimentais deveriam atender, ou seja, do lado direito; interessante notar que houve confirmação de uma pequena facilitação contra-lateral, mas de menor intensidade que no experimento III. 


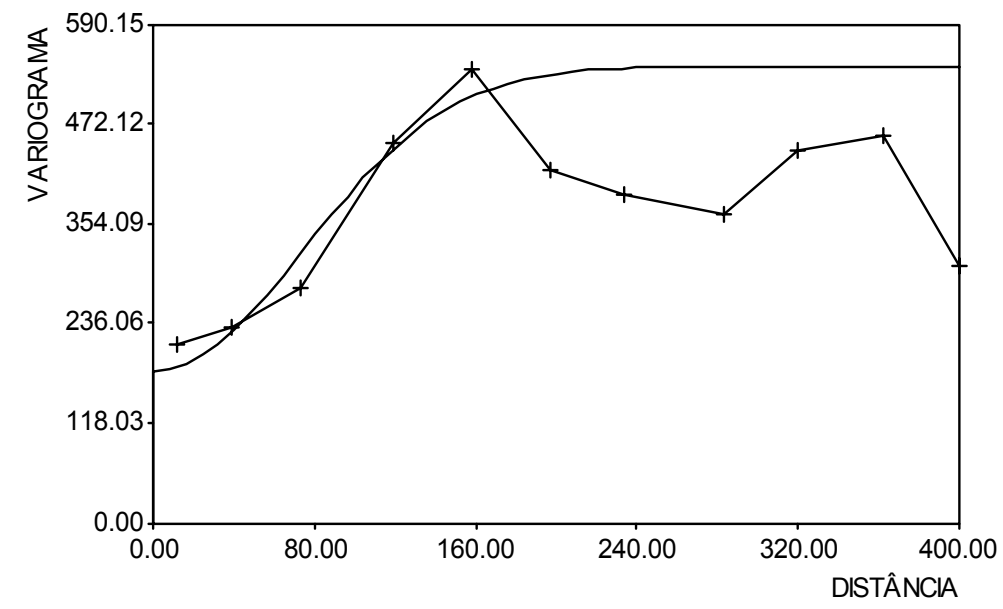

Figura 11: Ajuste do semivariograma do experimento IV, o ajuste foi realizado utilizando-se o modelo Gaussiano.



Figura 12: Mapa de pixeis do experimento IV, note-se o deslocamento do holofote atencional para o lado direito e, de maneira quase simétrica ao experimento III também uma pequena facilitação contra-lateral, mas de menor intensidade. 


\subsection{Experimento V}

Experimento de atenção dividida (focos atencionais tanto do lado esquerdo como do lado direito, simultaneamente); nesse experimento o ajuste do semivariograma (Figura 13) foi realizado utilizando-se o modelo esférico. Pode-se verificar que há uma clara divisão da atenção, com dois focos distintos, e com menores recursos atencionais na região intermediária (Figura 14).

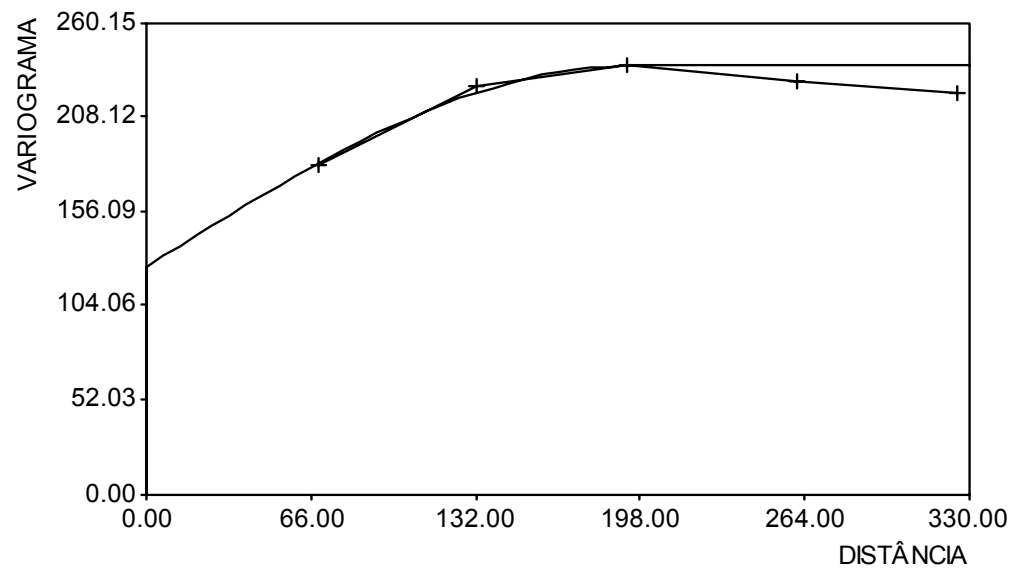

Figura 13: Ajuste do semivariograma do experimento V, o ajuste foi realizado utilizando-se o modelo esférico. 

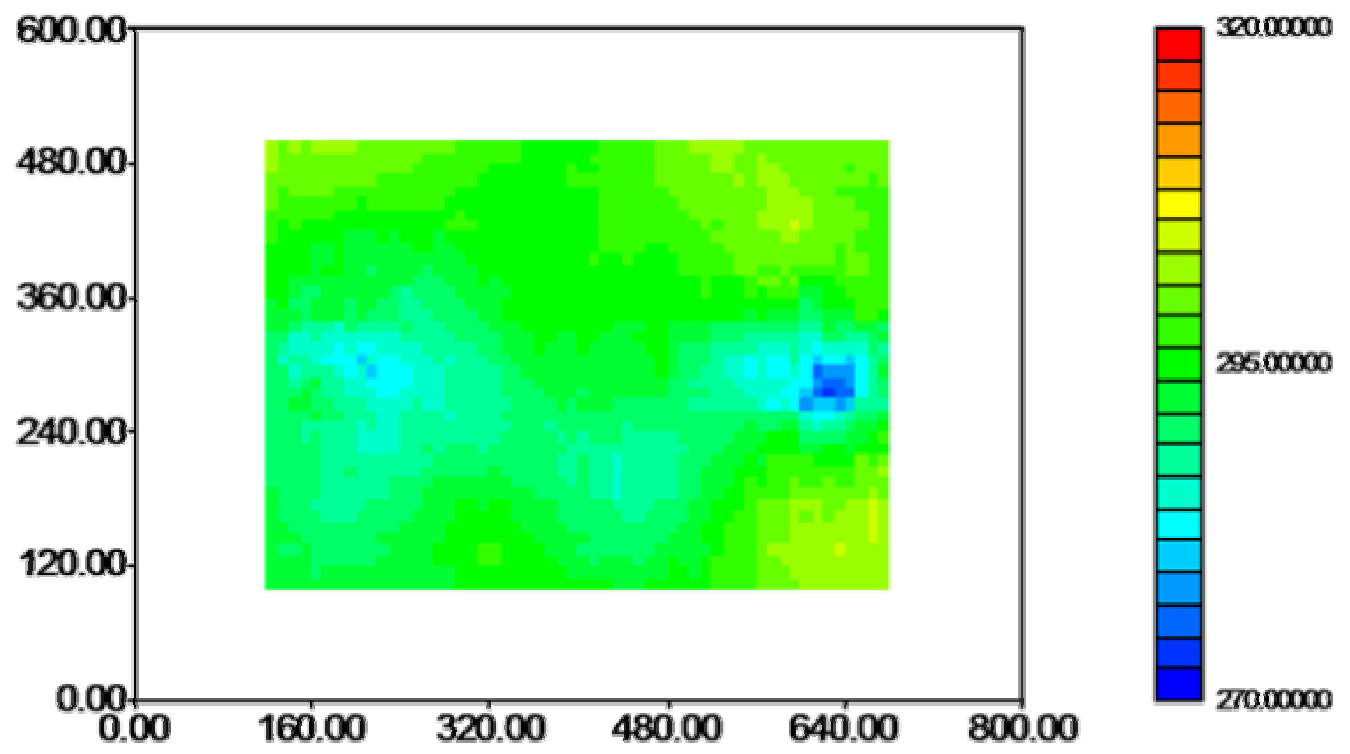

Figura 14: Mapa de pixeis do experimento $V$, aqui pode-se verificar que há uma clara divisão da atenção, com dois focos distintos, e com menores recursos atencionais na região intermediária. 


\section{DISCUSSÃO}

Os resultados acima apresentados demonstraram claramente a capacidade de desvincular a atenção do ponto de fixação e de alocar a atenção visual na região do espaço solicitada, seja ela na região central, no lado esquerdo, ou no lado direito, e simultaneamente nos lados esquerdo e direito (experimentos II, III, IV e V, respectivamente). Esses resultados foram apresentados em congresso, com publicação dos resumos, vide Anexo 4.

No presente trabalho, através do protocolo utilizado, e da análise geoestatística, foi possível verificar que:

- há um favorecimento do hemicampo inferior (experimento I), dado esse coerente com a literatura (relatos psicofísicos de uma anisotropia horizontal-vertical: ROVAMO e VIRSU, 1979; REGAN e BEVERLEY, 1983; KRÖSE e JULESZ, 1989; EDGARD e SMITH, 1990; PREVIC, 1990). Essa assimetria é coerente com a importância de atender, para o manuseio e análise, a objetos próximos, situação desse experimento. Possíveis correlatos neurais para essa assimetria são as maiores densidades de cones e células ganglionares no hemicampo visual inferior quando comparado ao hemicampo visual superior (PERRY e COWEY, 1985). Além disso uma maior área é dedicada ao hemicampo visual inferior do que ao hemicampo visual superior no Núcleo Geniculado Lateral (CONNOLLY e VAN ESSEN, 1984) e no córtex visual primário (VAN ESSEN et al., 1984; TOOTELL et al., 1988; TOOTELL et al. 1998).

- o holofote atencional, quando o voluntário foca a atenção no ponto foveado (experimento II), tem uma forma alongada com relação de 2:1 nos eixos horizontal e vertical; com características espaciais semelhantes à densidade de 
fotorreceptores (cones) na região de fóvea humana descrita por Curcio et al. (1990), apresentando-se ligeiramente deslocado para o hemicampo inferior. Estudos anatômicos e fisiológicos, realizados em macacos, também demonstram um possível correlato neural para essa anisotropia. Ou seja, há uma menor densidade de células ganglionares na retina (CURCIO e ALLEN, 1990) e uma maior queda de densidade de fotorreceptores (cones), indo da fóvea para a periferia no meridiano vertical do que no plano horizontal (CURCIO et al., 1987).

- na situação de atenção encoberta demonstramos mais uma vez a possibilidade de deslocamento do "holofote atencional", desvinculando o foco de atenção do ponto foveado. Os resultados dos experimentos III e IV mostram claramente esse deslocamento. Além disso podemos notar uma assimetria quando comparamos os mapas de pixeis dos experimentos III e IV. Essa assimetria é coerente com a encontrada por Kraft et al. (2005) e por Weber et al. (2005), sugerindo uma predominância do hemisfério cerebral direito. Nossos resultados também corroboram, se bem que apenas em parte, o modelo do "chapéu mexicano" proposto recentemente por Müller et al. (2005); isto porque encontramos uma facilitação contra-lateral simétrica ao foco atencional, e não uma borda anular ao redor do foco central como o proposto por esse modelo. Essa facilitação contralateral está presente nos dados de Martínez et al. (1999) em um estudo eletrofisiológico, porém esses autores não comentam o achado;

- os resultados do experimento $\mathrm{V}$ demonstram a possibilidade de haver divisão atencional, como sugerido por Awh e Pashler (2000), Muller et al. (2003) e Kraft et al. (2005). Nossos resultados, porém, além de confirmarem a possibilidade de haver divisão atencional, detalham tal divisão de forma muito mais clara e quantitativa. 
Além da análise geoestatística empregada na seção Resultados, aplicamos, na mesma base de dados, um outro método geoestatístico, qual seja a simulação estocástica, que tem como principal característica à de fornecer informações relativas ao comportamento global da variável de interesse, ao contrário da krigagem ordinária que fornece maior precisão local. Através dessa análise podemos verificar (Anexo 5) um padrão similar ao encontrado pela análise com krigagem ordinária.

Visto o sucesso do método e o interesse dos resultados obtidos, foi possível demonstrar algumas aplicações, que apenas demonstram as perspectivas abertas pelo método:

1. verificar se as assimetrias encontradas no experimento I, ou seja, o favorecimento atencional do quadrante inferior esquerdo (nasal), seria confirmado estudando o olho contralateral. No Anexo 6 são apresentados resultados que confirmam essa assimetria, situando-a no quadrante inferior nasal do campo visual (ou seja no quadrante inferior direito). Esse achado fortalece a discussão acima, que foca a importância da alocação da atenção nesse quadrante para o manuseio de objetos e utensílios na nossa espécie.

2. tentar segregar efeitos atencionais nas vias visuais ventral e dorsal. Para isso, propusemos, em colaboração com colegas da Universidade de Roma "La Sapienza", que os experimentos acima descritos, com medidas de tempos de reação simples, estariam todos envolvendo prioritariamente a via dorsal, apelidada de "via aonde", e que essa seria a razão para o favorecimento do hemicampo visual inferior. Tempos de reação simples seriam mais envolvidos com o local do que com qualquer outra característica do estímulo. Para estudar a via ventral, apelidada de "via o quê”, foi adotado um protocolo com medidas de tempos de reação escolha. 
Em tal protocolo, há duas possibilidades de estímulos a serem apresentados aos voluntários: uma cruz ou um círculo. Se aparecer uma cruz, o voluntário deveria apertar uma dada tecla, mas se aparecer um círculo, outra tecla deveria ser apertada pelo voluntário. Resultados relativos à atenção difusa, Experimento I, são apresentados no Anexo 7, indicando uma diferença notável da distribuição da atenção nos dois casos; para tempos de reação escolha, de fato, não há privilégio do hemicampo inferior;

3. verificamos, em colaboração com colegas da Universidade Federal Fluminense, especialistas da área, o efeito da intoxicação aguda por álcool sobre a distribuição da atenção. Obtivemos resultados muito interessantes, mostrando que intoxicação aguda por álcool impede a desvinculação da atenção do ponto foveado (Anexo 8). Há interesse de ordem prática nessa observação, considerando a importância da atenção no guiar veículos ou operar máquinas após o consumo de álcool. Esse trabalho foi enviado e aceito para publicação (Anexo 9);

4. dando seqüência ao trabalho acima, verificamos o efeito da dose de álcool nos padrões de distribuição da atenção; para tanto realizamos experimentos com quatro diferentes doses (além de placebo e controle). Muito interessantes são as perspectivas de aplicação a outras drogas psicoativas, como antidepressivos. A caracterização do efeito dessas drogas em termos atencionais abre uma perspectiva motivadora e inédita de aplicações clínicas de nosso protocolo, que possui também a vantagem de ser um teste relativamente rápido (4-5 minutos por teste) em comparação a métodos psicofísicos clássicos. 


\section{CONCLUSÃO}

Com base nos nossos achados, podemos concluir que:

I. O método geoestatístico é eficaz em aplicações psicofísicas.

II. Há um favorecimento de hemicampo inferior na situação de atenção difusa.

III. É possível direcionar a atenção de forma explícita, encoberta e também de dividir a atenção. 


\section{REFERÊNCIAS}

AWH, E.; PASHLER, H. Evidence for split attentional foci. J. Exp. Psychol. Hum. Percept. Perform., v. 26, p. 834-846, 2000.

BACH, M. The "Freiburg Visual Acuity Test" - Automatic measurement of visual acuity. Optometry and Vision Science, v. 73, p. 49-53, 1996.

BALDASSI, S.; BURR, D. C. Feature-based integration of orientation signals in visual search. Vision Research, v. 40, p. 1293-1300, 2000.

BREFCZYNSKI; J. A.; DEYOE, E. A. A physiological correlate of the 'spotlight' of visual attention. Nature Neuroscience, v. 2, n. 4, p. 370-374, 1999.

CARRASCO, M.; MCELREE, B. Covert Attention accelerates the rate of visual information processing. Proceedings of the National Academy of Sciences, v. 98, p. 5363-5367, 2001.

CARRASCO, M.; TALGAR, C. P.; CAMERON, E. L. Characterizing visual performance fields: effects of transient covert attention, spatial frequency, eccentricity, task and set size. Spatial Vision, v. 15, p. 61-75, 2001.

CARRASCO, M.; WILLIAMS, P. E..; YESHURUN, Y. Covert attention increases spatial resolution with or without masks? Support for signal enhancement. Journal of Vision, v. 2, p. 467-479, 2002.

CASTIELLO, U.; UMILTA, C. Size of the attentional focus and efficency of processing. Acta Psychol., v. 73, p. 105-113, 1990.

CONNOLLY, M.; VAN ESSEN, D. The representation of the visual field in parvocellular and magnocellular layers of the lateral geniculate nucleus in the macaque monkey. J. Comparative Neurol., v. 266, p. 544-564, 1984.

CURCIO, C. A.; SLOAN, K. R.; PACKER, O.; HENDRICKSON, A. E.; KALINA, R. E. Distribution of cones in human and monkey retina: Individual variability and radial asymmetry. Science, v. 236, p. 579-582, 1987.

CURCIO, C. A.; ALLEN, K. A. Topography of ganglion cells in human retina. J. Comparative Neurol., v. 300, n. 1, p. 5-25, 1990.

CURCIO, C. A.; SLOAN, K. R.; KALINA, R. E.; HENDRICKSON, A. E. Human photoreceptor topography. The Journal of Comparative Neurology, v. 292, p. 497 523, 1990.

DOWNING, C. J.; PINKER, S. The spatial structure of visual attention. In M. I. Posner \& O. S. M. Marin (Eds.). Attention and performance XI: Mechanisms of attention. Hillsdale: Erlbaum, 1985. 675p. 
EDGARD, G. K.; SMITH, A. T. Hemifield differences of perceived spatial frequency, Perception, v. 19, p. 759-766, 1990.

EIMER, M. Attending to quadrants and ring-shaped regions: EPR effects of visual attention in different spatial selection tasks. Psychophysiology, v. 36, p. 491-503, 1999.

ERIKSEN, C. W.; YEH, Y. Y. Allocation of attention in the visual field. J. Exp. Psychol. Hum. Percept. Perfom, v. 11, p. 583-597, 1985.

ERIKSEN, C. W.; ST. JAMES, J. D. Visual attention within and around the field of focal attention: A zoom lens model. Perception and Psychophysics, v. 40, p. 225240, 1986.

FUCHS, K.; DEUTZ, A. Use of variograms to detect critical spatial distances for the Knox's test. Preventive Veterinary Medicine, v. 54, p. 37-45, 2002.

GOBELL, J. L.; CHIA-HUEI, T.; SPERLING, G. The spatial distribution of visual attention. Vision Research, v. 44, p. 1273-1296, 2004.

GOOVAERTS, P. Geostatistics for natural resources evaluation. New York: Oxford University Press, 1997. 496 p.

JOURNEL, A. G. Geostatistics: models and tools for the earth sciences. Math. Geol., v. 18, p. 119-140, 1979.

HEINZE, H.; LUCK, S. J.; MUENTE, T. F.; GOES, A.; MANGUN, G. R.; HILLYARD, S. A. Attention to adjacent and separate positions in space: An electrophysiological analysis. Perception and Psychophysics, v. 56, p. 42-52, 1994.

KRAFT, A.; MÜlLER, N. G.; HAGENDORF, H.; SCHIRA, M. M.; DICK, S.; FENDRICH, R. M.; BRANDT, S. A. Interactions between task difficulty and hemispheric distribution of attended locations: implications for the splitting attention debate. Cognitive Brain Research, v. 24, p. 19-32, 2005.

KRAMER, A. F.; HAHN, S. Splitting the beam: distribution of attention over noncontiguous regions of visual field. Psychological Science, v. 6, p. 381-386, 1995.

KRÖSE, B. J. A.; JULESZ, B. The control and speed of shifts of attention, Vision Research, v. 29, p. $1607-1619,1989$.

LUDOVISIA, A.; MINIZZOB, M.; PANDOLFIA, P.; TATICCHIA, M. I. Modelling the horizontal spatial structure of planktonic community in Lake Trasimeno (Umbria, Italy) using multivariate geostatistical methods. Ecological Modelling, v. 181, p. 247$262,2005$.

MARTÍNEZ, A.; ANLLO-VENTO, L.; SERENO, M. I.; FRANK, L. R.; BUXTON, R. B.; DUBOWITZ, D. J.; WONG, E. C.; HIRICHS, H.; HEINZE, H. J.; HILLYARD, S. A. Involvement of striate and extrastriate visual cortical areas in spatial attention. Nat. Neurosci., v. 2, p. 364-369, 1999. 
MATHER, F. J.; WHITE, L. E.; LANGLOIS, E. C.; SHORTER, C. F.; SWALM, C. M.; SHAFFER, J. G.; HARTLEY, W. R. Statistical methods for linking health, exposure, and hazards. Environmental Health Perspectives, v. 112, n. 14, p. 1440$1445,2004$.

MIRANDA, M. L.; DOLINOY, D. C. Using GIS-Based approaches to support research on neurotoxicants and other children's environmental health threats. Neurotoxicology, v. 26, p. 223-228, 2005.

MÜLLER, M. M.; MALINOWSKI, P.; GRUBER, T.; HILLYARD, S. A. Sustained division of the attentional spotlight. Nature, v. 424, p. 309-312, 2003.

MÜLLER, N. G.; MOLLENHAUER, M.; RÖSLER, A.; KLEINSCHMIDT, A. The attentional field has a Mexican hat distribution. Vision Research, v. 45, p. 1129$1137,2005$.

PAN, K.; ERIKSEN, C. Attentional distribution in the visual field during samedifferent judgments as assessed by response competition. Perception \& Psychophysics, v. 53, p. 134-144. 1993.

PERRY, V. H.; COWEY, A. The ganglion cell and cone distribution in the monkey's retina: Implications for central magnification factors. Vision Research, v. 25, p. 1795-1810, 1985.

POSNER, M. I. Orienting of attention. Quarterly Journal of Experimental Psychology, v. 32, p. 3-25, 1980.

POSNER, M. I.; DAVIDSON, B. J.; SNYDER, C. R. R. Attention and the detection of signals. Journal of Experimental Psychology: General, v. 109, n. 2, p. 160-174, 1980 .

POSNER, I. P.; PETERSEN, S. E. The attention system of the human brain. Annu. Rev. Neurosci., v. 13, p. 25-42, 1990.

PREVIC, F. H. Functional specialization in the lower and upper visual fields in humans: Its ecological origins and neurophysiological implications, Behavioral and Brain Sciences, v. 13, p. 519-575, 1990.

REAGAN, D.; BEVERLEY, K. I. Visual fields described by contrast sensitivity, by acuity, and by relative sensitivity to different orientations. Investig. Ophthalmol. Visual Science, v. 24, p. 754-759, 1983.

ROVAMO, J.; VIRSU, V. An estimation and application of the human cortical magnification factor, Experimental Brain Research, v. 37, p. 495-510, 1979.

SCHNEIDER, W.; ESCHMAN, A.; ZUCCOLOTTO, A. E-Prime User's Guide. Pittsburgh: Psychology Software Tools Inc., 2002a. 208 p. 
SCHNEIDER, W.; ESCHMAN, A.; ZUCCOLOTTO, A. E-Prime Reference Guide. Pittsburgh: Psychology Software Tools Inc., 2002b. 220 p.

SEGALOWITZ, S. J.; GRAVES, R. Suitability of the IBM XT, AT and PS/2 keyboard, mouse, and game port as response devices in reaction time paradigms. Behavior Research Methods, Instruments, and Computers, v. 22, p. 283-289, 1990.

SHULMAN, G. L.; SHEEHY, J. B.; WILSON, J. Gradients of spatial attention. Acta Psychologica, v. 61, p. 167-181, 1986.

TOOTELL, R. B. H.; SWITKES, E.; SILVERMAN, M. S.; HAMILTON, S. L. Functional anatomy of macaque striate cortex. II. Retinotopic organization. J.

Neurosci., v. 8, n. 5, p. 1531- 1568, 1988.

TOOTELL, R. B. H.;HADJIKHANI, N.; HALL, E. K.; MARRET, S.;

VANDUFFEL, W.; VAUGHAN, J. T.; DALE, A. M. The retinotopy of visual spatial attention. Neuron, v. 21, p. 1409-1422, 1998.

VAN ESSEN, D. C.; NEWSOME, W. T.; MAUNSELL, J. H. R. The visual field representation in striate cortex of the macaque monkey: Asymmetries, anisotropies, and individual variability. Vision Research, v. 24, n. 5, p. 429-448, 1984.

YAMAMOTO, J. K. An Alternative measure of the reliability of ordinary kriging estimates. Math. Geology, v. 32, p. 489-509, 2000.

YAMAMOTO, J. K. Sistema GeoVisual - aplicativos em Delphi para tratamento geoestatístico de dados espaciais. 2002.

WEBER, B.; TREVER, V.; OBERHOLZER, N.; JAERMANN, T.; BOESIGER, P.; BRUGGER, P.; REGARD, M.; BUCK, A.; SAVAZZI, S.; MARZI, C. A. Attention and interhemispheric transfer: A behavioral and fMRI study. Journal of Cognitive Neuroscience, v. 17, p. 113-123, 2005. 
ANEXOS 
Anexo 1

- Termo de Consentimento Livre e Esclarecido. 


\section{TERMO DE CONSENTIMENTO LIVRE E ESCLARECIDO}

\section{ESTUDO: Mapeamento espacial da atenção através de tempos de reação: um estudo psicofísico}

Você está sendo convidado(a) a participar do projeto de pesquisa acima citado. $O$ documento abaixo contém todas as informações necessárias sobre a pesquisa que estamos fazendo. Sua colaboração neste estudo será de muita importância para nós, mas se desistir a qualquer momento, isso não causará nenhum prejuizo a você.

$\mathrm{Eu}$,

residente e domiciliado(a) na , portador da cédula de identidade, $\mathrm{RG} \mathrm{n}^{\circ}$

$\longrightarrow$ e inscrito no $\mathrm{CPF} / \mathrm{MF}$ sob $\mathrm{n}^{\circ}$ nascido(a) em ___ _ _ _ abaixo assinado(a), concordo de livre e espontânea vontade em participar como voluntário(a) do estudo “Mapeamento espacial da atenção através de tempos de reação: um estudo psicofísico". Declaro que obtive todas as informações necessárias, bem como todos os eventuais esclarecimentos quanto às dúvidas por mim apresentadas.

Estou ciente que:

I) $\quad \mathrm{O}$ estudo se faz necessário para que se possam estudar as possíveis interações da atenção voluntária sobre os tempos de reação e seu conseqüente mapeamento espacial;

II) Serão feitos cinco experimentos com duração aproximada de 30 minutos no total;

III) Tenho a liberdade de desistir ou de interromper a colaboração neste estudo no momento em que desejar, sem necessidade de qualquer explicação;

IV) A desistência não me causará nenhum prejuízo;

V) Os resultados obtidos durante este trabalho de pesquisa serão mantidos em sigilo, mas concordo que sejam divulgados em publicações científicas, desde que meus dados pessoais não sejam mencionados;

VI) Caso eu desejar, poderei pessoalmente tomar conhecimento dos resultados, ao final desta pesquisa 
( ) Desejo conhecer os resultados desta pesquisa.

( ) Não desejo conhecer os resultados desta pesquisa.

São Paulo, de de 200

Voluntário :

Telefone para contato:

E-mail :

Testemunha 1 :

Nome / RG / Telefone

Testemunha 2 :

Nome / RG / Telefone

Responsável pelo estudo:

Telefone para contato: 3091-7215

Luiz Henrique M. do Canto Pereira

E-mail : canto@usp.br 
Anexo 2

- Anamnese. 
ESTUDO: Mapeamento espacial da atenção através de tempos de reação: um

$$
\text { estudo psicofísico }
$$

\section{ANAMNESE}

NOME:

END.:

CIDADE:

EST.:

TEL.:

DATA DE NASC: NATURALIDADE:

Apresenta algum problema de visão? ( ) SIM NÃO

Se SIM - Qual o problema e quando começou?

Se NÃO - Há alguém na família que apresente? Quem e qual o problema?

Faz algum tratamento / usa óculos?

Apresenta algum problema de saúde?

1- Diabetes mellitus ( ) SIM N ( ) NÃO

Se SIM - Qual o tipo? Há quanto tempo?

Se NÃO - Há alguém na família que apresente? Quem e que tipo?
2- Hipertensão arterial sistêmica
( ) SIM
( ) NÃO

Se SIM - Há quanto tempo?

Se NÃO - Há alguém na família que apresente?

3- Já foi submetido a alguma procedimento cirúgico? ( ) SIM N ( ) NÃO

Se SIM - Qual? Quando?

4- Já foi vítima de algum acidente que tenha atingido alguma região da cabeça?

5- Outras informações relevantes: 
INTERROGATÓRIO COMPLEMENTAR

Tabagista? ( ) SIM ( ) NÃO

Se SIM - Que tipo ?

Há quanto tempo? Que quantidade?

Etilista? ( ) SIM NÃO

Se SIM - Que tipo de bebida?

Há quanto tempo? Que quantidade?

Usuário de drogas? ( ) SIM ( ) NÃO

Se SIM - Que tipo?

Há quanto tempo? Que quantidade?

Faz uso de algum medicamento frequentemente? ( ) SIM $\quad$ ( ) NÃO

Se SIM - Qual?

Exerceu alguma atividade que o mantivesse em contato com substâncias químicas? ( )SIM ( )NÃO Se SIM: Qual atividade?

Que tipo de substância?

Qual a frequência de exposição?

Apresentou algum sintoma diferente durante esta atividade?

AVALIAÇÕES COMPLEMENTARES:

Acuidade visual olho dominante

O.D.

O.E.

Mão dominante

M.D.

M.E.

Declaro que fui informado dos objetivos do presente estudo, ter consentido com minha participação e haver preenchido o Termo de Consentimento Livre e Esclarecido em anexo.

São Paulo, de de 200

Voluntário :

Responsável: 
Anexo 3

- Trabalho apresentado no "XXth Annual Meeting of the International Society for Psychophysics - Fechner's Day 2004” em Coimbra, Portugal.

- Trabalho publicado no Proceedings of the XXth Annual Meeting of the International Society for Psychophysics - Fechner's Day 2004. p. 332-337. 


\title{
MAPPING THE SPATIAL DISTRIBUTION OF ATTENTION THROUGH REACTION TIMES
}

\author{
Canto Pereira, Luiz Henrique M. ; Ranvaud, Ronald D.
}

\author{
Laboratório de Fisiologia do Comportamento - Departamento de Fisiologia e Biofísica -Instituto de Ciências Biomédicas I \\ Núcleo de Neurociências e Comportamento - Universidade de São Paulo - Brazil
E-mail: canto@usp.br
}

\section{INTRODUCTION}

In the past twenty years visual attention has been described in terms of varied metaphors, among which a spotlight (Posner, 1980), a zoom lens (Ericksen and St.James, 1986), and a gradient field (LaBerge, 1995; Downing and which is not necessarily determined by the fixation point, and may depend on both external events and internal which is not An essanly demin mut a processes. Reaction visual field with this very simple technique.
vistigate attentional allocation covering large continuous areas of the

In the present study we propose a new approach to analyze data using a geostatistics method. This technique, which is widely used in values which are farther away from each other. The basic concept of geostatistics is that of scales of spatial variation.

\section{METHOD}

\section{Subjects}

Graduate and undergraduate students $(n=10)$ from the University of Sao Paulo, ( 2 males and 8 females), aged 19 40 (mean $27.1 \pm 6.8$ ) participated in the experiments. Inclusion criteria were: $20 / 25$ Snellen best-corrected visual acuity or better; absence of known ophthalmological pathologies; no previous experience with psychophysical experiments. The experiments were performed after all subjects had received adequate information on the experiment and had signed their informed consent.

Equipment

Reaction times (RTs) were presented and measured with the software E-Prime v 1.1 (PST Inc.) using a 17' monitor driven by a Matrox $\mathrm{P} 650$ graphics board with a refresh rate of $100 \mathrm{~Hz}$ and a resolution of $800 \times 600$. From the subjects' viewing distance $\left(57 \mathrm{~cm}\right.$ ) the screen subtended $32^{\circ} \times 24^{\circ}$ of visual angle. The subjects' responses were registered with millisecond accuracy through a joystick connected to the game port of the PC. The subjects were positioned in front of the monitor and maintained in this position during the experiment with their heads stabilized by a chin rest. An infrared camera was used to monitor eye movements during the experiments, and trials were discarded if the eye deviated more than $1^{\circ}$ from the fixation point


Procedure

* All experimental sessions were performed in a darkened, sound attenuated booth. Participants viewed stimuli with their dominant eye, and responded by pressing a button with their dominant hand. Prior to data collection, subjects went through a training session allowing them to become acquainted with the procedure. Subjects were required to keep their gaze on a small cross in the center of the computer screen. The visual stimulus to which subjects had to respond as quickly as possible (simple reaction time) was a white dot, subtending $0.2^{\circ}$ of visual angle, with a luminance of $80 \mathrm{~cd} / \mathrm{m}^{2}$, against a gray background with luminance of $20 \mathrm{~cd} / \mathrm{m}^{2}$ presented at different positions on the computer screen. Stimulus duration was brief $(100 \mathrm{~ms})$ to avoid eye movements and concomitant attentional shifts. RTs below $150 \mathrm{~ms}$ and above $500 \mathrm{~ms}$ were considered invalid and in both cases were replaced with a now * In experiment 1 participants were asked not to attend to any particular region, but rather try to spread their attention as uniformly as possible over the computer screen (diffuse attention). In the remaining experiments, participants were ixstructed to direct their visual attention coverty to the center (experiment 2), to the left (experiment 3 ), to the right (experiment 4) or to both right and left (but not to the center, thus characterizing a divided attention situation - experiment 5). Figure 2 shows the positions where the stimuli were presented. The grid subtended $22.4^{\circ}$ by $16^{\circ}$ of visual angle, and each point in the grid was used only once in each experiment.

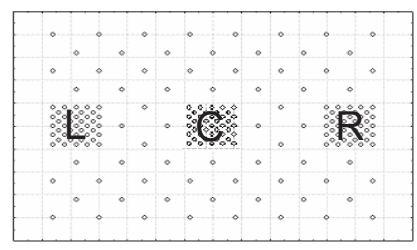

Figure 2: Grid of positions where stimuli were presented in the 5 experiments. The sparsely distributed points covering
the whole region were presented in all experiments. In addition, in experiments $2,3,4$ and 5 the densely distribute the whole region were presented in all experiments. In addititon, in experiments $2,3,4$ and 5 the densely distributee
points in the squares marked $C, L, R$ and $(L+R)$ were added, respectively. $C$ stands for center, $L$ for left and $R$ for right

Data Analysis

RTs were graphically presented as contour plots which show where subjects were quicker or slower (software Idrisi - Kilimanjaro v. 14.2 - Clark Labs - with Gstat geostatistical module). The RTs were also submitted to a oneway analysis of variance (ANOVA) with Tukey's post hoc pairwise comparisons (Minitab Statistical Software release 13.0). The statistical significance level used was $p<0.05$

\section{RESULTS}

* Statistical analysis (one-way ANOVA) showed that the mean RT of experiment 2 was faster than that of experiments 3,4 and $5(F=11.56, p<0.001)$ as shown in Figure 3 . There were also marginal differences between the mean RT of experiments 1 and 3,5 .

- The distribution of RTs in the visual field of the subjects in experiments 1,2,3,4 and 5 are shown in Figures 4A, 4B, $4 \mathrm{C}, 4 \mathrm{D}$ and $4 \mathrm{E}$ respectively. Shorter or longer RTs were taken to indicate, respectively, higher or lower attentiona focus. The contour plots show much structure, superposed on which there is a general tendency towards shorter times to the left in experiment 3 , to the right in experiment 4 , and distributed to both right and left (even though not as lateralized as in the previous two experiments) in experiment 5 .

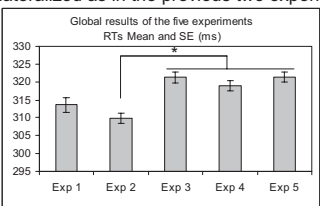

Figure 3: Data (RTs in ms) from experiments 1 to 5 (mean and S.E).

(") Indicates statistically significant different
experiment 2 and experiments 3.4 and 5 .



\section{DISCUSSION}

The present study indicates that in spite of the variability of RTs (much larger than the effects of eccentricity in all of the experimental conditions here investigated), some qualitative indication of the distribution of visual attentio Figure 2 was characterized by just ten RTs in the five experiments (Fig 4). The present results encourage increasing the sample size to obtain clearer images of attentional distributions, with higher spatial resolution and less noise. There is some indication that the center of the visual field may be somewhat attended at all times, even when subjects were instructed to pay attention to the right or to the left of center. When they were required to attend both to the right and to the left, they seemed to have some difficulty in separating the two foci. This was both evident from the lower eccentricity of the regions with fast RTs in experiment 5 when compared to experiments 3 and 4 , and from residual fast $R T$ in the central area in both experiments 3 and 4 . Still, at least qualitatively, Figs $4 A-E$ have the broad ceparate arcas at the same time, at least with the present experimental design. Acknowedgments: We are graful to CNPq (Gant \# 141951/20 Acknowledgments: We are grata from 


\title{
MAPPING THE SPATIAL DISTRIBUTION OF ATTENTION THROUGH REACTION TIMES
}

\author{
Luiz H. M. Canto-Pereira, Ronald D. Ranvaud \\ Departamento de Fisiologia e Biofísica, Instituto de Ciências Biomédicas, \\ Núcleo de Neurociências e Comportamento \\ Universidade de São Paulo, São Paulo, Brasil \\ E-mail: canto@usp.br
}

\begin{abstract}
The present study aimed at mapping the spatial distribution of attention in five different experimental situations using simple reaction times. Subjects $(n=10)$ were asked to spread their attention uniformly over a $22.4^{\circ}$ by $16^{\circ}$ frontal region of the visual field, or to orient their voluntary covert attentional focus towards one or two of 3 possible areas within this region, while always maintaining their gaze on a centrally located small cross. In experiment 1 (diffuse attention situation) stimulus probability was uniform over the region of interest, sampled at 82 evenly spaced points, and subjects were instructed not to attend to any particular area; in experiment 2 subjects were asked to orient their visual attention at the central area of the computer screen, at and immediately around their fixation point; in experiment 3 they were asked to attend an area to the left of center; in experiment 4 to the right of center; and in experiment 5 to two separate areas, one to the right and the other to the left (divided attention situation). Mean reaction times differed significantly among experiments, being faster in experiment 2 (central focus situation). Reaction times also tended to be faster in or near the attended areas in all experiments. The great variability in reaction times require quite large sample sizes to overcome noise, but the results thus far obtained encourage further applications of the experimental protocol.
\end{abstract}

In the past twenty years visual attention has been described in terms of varied metaphors, among which a spotlight (Posner, 1980), a zoom lens (Ericksen and St.James, 1986), and a gradient field (LaBerge, 1995; Downing and Pinker, 1985). An essential aspect of all these metaphors is the distribution of attentional resources in the visual field, which is not necessarily determined by the fixation point, and may depend on both external events and internal mental processes. A much-debated question is whether the flexibility of attentional distribution in space includes the possibility of attending two separate areas, to the exclusion of the space between them. Awh and Pashler (2000), for example, present 
recent evidence in favour of the possibility of split attentional foci, whereas several prior experiments seemed to question this possibility (Eriksen and Yeh, 1985; Pan and Eriksen, 1993; Heinze et al. 1994; Eimer, 1999). The latest evidence in favour of the possibility of two spatially separate attentional foci comes from Müller et al. (2003), who recorded evoked potentials in the visual primary cortex of human subjects, showing elegant isocontour maps of cortical activity as the subjects were exposed to different stimuli.

Gobell et al. (2004) went a step further, requiring subjects to attend to alternate stripes while strongly ignoring the intervening stripes to perform a search task. These authors thus not only investigated the possibility of dividing the attentional focus, but also covered a large continuous region of the visual field by presenting visual stimuli over the whole surface of interest. Prior work on divided attention only sampled isolated, specific areas of the visual field. Gobell et al.(2004) further propose a general theoretical model with which to calculate how attention would be distributed under any stimulus configuration, through Fourier analysis.

Reaction times have been at the basis of several investigations concerning attentional allocation, but apparently no one attempted thus far to investigate attentional allocation covering large continuous areas of the visual field with this very simple technique. The present contribution presents an attempt to do so, and indicates that, in spite of the need to collect rather large data sets, it is possible to obtain interesting information about how visual attention is allocated in continuous regions of space by measuring the reaction time of subjects under different task requirements. The data here presented indicates that there appears to be indeed some difficulty in splitting attentional focus, but that this possibility is not excluded.

\section{Method}

Subjects. Graduate and undergraduate students $(\mathrm{n}=10)$, from Instituto de Ciências Biomédicas of University of Sao Paulo, (2 males and 8 females), aged 19-40 (mean $27.1 \pm 6.8$ ) participated in the present study. Inclusion criteria were: $20 / 25$ Snellen bestcorrected visual acuity or better; absence of known ophthalmological pathologies; no previous experience with psychophysical experiments. The experiments were performed after all subjects had received adequate information on the experiment and had signed their informed consent. The procedures followed the tenets of the Declaration of Helsinki.

Equipment. Reaction times (RTs) were measured with the software E-Prime v 1.1 (PST Inc.) (Schneider et al. 2002) using a 17' Samsung 753DFX monitor powered by a PC Athlon XP 2400/512 driven by a Matrox P650 graphics board with a refresh rate of $100 \mathrm{~Hz}$ and a resolution of $800 \times 600$. From the subjects' viewing distance $(57 \mathrm{~cm})$ the screen subtended $32^{\circ} \times 24^{\circ}$ of visual angle. The subjects' responses were registered with millisecond accuracy (Segalowitz and Graves, 1990) through a joystick connected to the game port of the PC. The subjects were positioned in front of the monitor and maintained in this position during the experiment with their heads stabilized by a chin rest. An infrared camera was used to monitor eye movements during the experiments, and trials were discarded if the eye deviated more than $1^{\circ}$ from fixation point 
Procedure. All experimental sessions were performed in a darkened, sound attenuated room. Subjects viewed stimuli with their dominant eye, and responded by pressing a button with their dominant hand. Prior to data collection, subjects went through a training session allowing them to become acquainted with the procedure. Subjects were required to keep their gaze on a small cross in the center of the computer screen. The visual stimulus to which subjects had to respond as quickly as possible (simple reaction time) was a white dot, subtending $0.2^{\circ}$ of visual angle, with a luminance of $80 \mathrm{~cd} / \mathrm{m}^{2}$, against a gray background with luminance of $20 \mathrm{~cd} / \mathrm{m}^{2}$ presented at different positions on the computer screen. Stimulus duration was brief $(100 \mathrm{~ms})$ to avoid eye movements and concomitant attentional shifts (Müller et al. 2003). RTs below $150 \mathrm{~ms}$ and above $500 \mathrm{~ms}$ were considered invalid and in both cases were replaced with a new trial.

In experiment 1 subjects were asked not to attend to any particular region, but rather try to spread their attention as uniformly as possible over the computer screen (diffuse attention). In the remaining experiments, subjects were instructed to direct their visual attention covertly to the center (experiment 2 ), to the left (experiment 3 ), to the right (experiment 4) or to both right and left, but not to the center, characterizing a divided attention situation (experiment 5). Figure 1 shows the positions where the stimuli were presented in the five experiments. The grid subtended $22.4^{\circ}$ by $16^{\circ}$ of visual angle, and each point in the grid was used only once in each experiment.

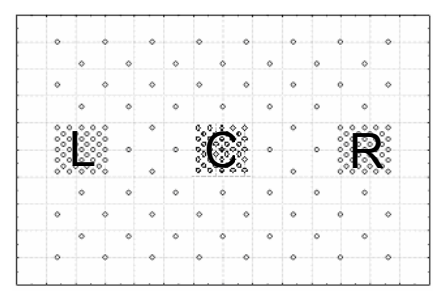

Figure 1: Grid of positions where stimuli were presented in the 5 experiments. The sparsely distributed points covering the whole region were presented in all experiments. In addition, in experiments 2, 3, 4 and 5 the densely distributed points in the squares marked $\mathrm{C}, \mathrm{L}, \mathrm{R}$ and $(\mathrm{L}+\mathrm{R})$ were added, respectively. $\mathrm{C}$ stands for center, $\mathrm{L}$ for left and $\mathrm{R}$ for right.

Data Analysis. RTs were graphically presented as contour plots (Minitab Statistical Software release 13.0) which show where subjects were quicker or slower. The RTs were also submitted to a one-way analysis of variance (ANOVA) with Tukey's post hoc pairwise comparisons. The statistical significance level used was $\mathrm{p}<0.05$.

\section{Results}


Statistical analysis (one-way ANOVA) showed that the mean RT of experiment 2 was faster than that of experiments 3,4 and $5(\mathrm{~F}=11.56, \mathrm{p}<0.001)$ as shown in Figure 2. There were also marginal differences between the mean RT of experiments 1 and 3, 5 .

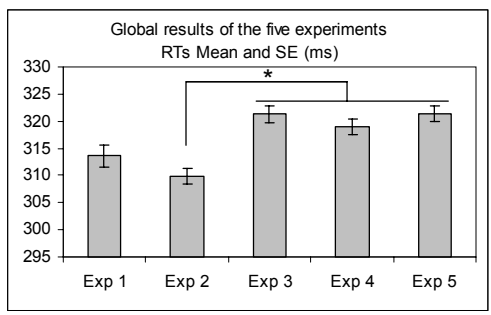

Figure 2: Data (RTs in ms) from experiments 1 to 5 (mean and S.E).

$\left({ }^{*}\right)$ Indicates statistically significant differences $(\mathrm{p}<0.001)$ between experiment 2 and experiments 3,4 and 5 .

The distribution of RTs in the visual field of the subjects in experiments 1,2,3,4 and 5 are shown in Figures 3,4,5, 6 and 7 respectively. Shorter or longer RTs were taken to indicate, respectively, higher or lower attentional focus. The contour plots show much structure, superposed on which there is a general tendency towards shorter times to the left in experiment 3 , to the right in experiment 4 , and distributed to both right and left (even though not as lateralized as in the previous two experiments) in experiment 5.

Contour Plot of Experiment 1

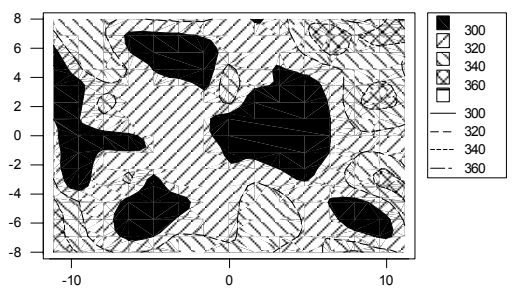

Figure 3: Contour plot of RTs obtained in experiment 1 , showing a diffuse pattern of attended regions.

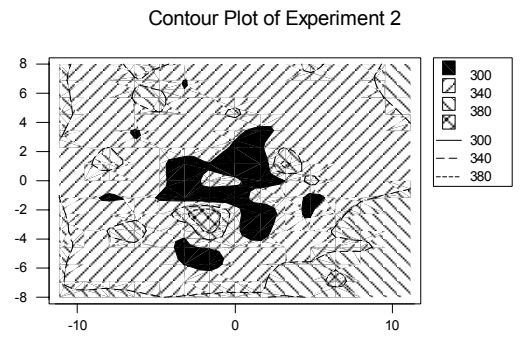

Figure 4: Contour plot of RTs obtained in experiment 2 showing a tendency for shorter RTs to congregate near the center, coincident with the attentional focus. 
Contour Plot of Experiment 3

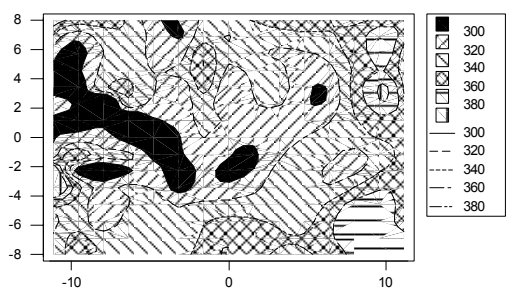

Figure 5: Contour plot of RTs obtained in experiment 3 showing clearly a shift to the left. A residual attended locus in the central region seems to be present.

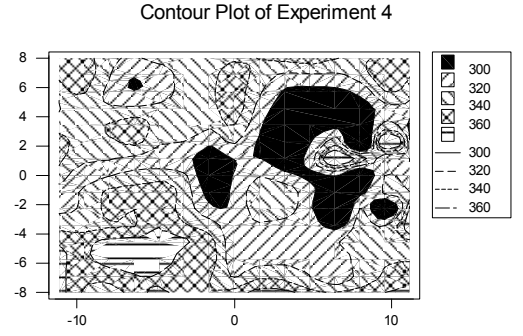

Figure 6: Contour plot of RTs obtained in experiment 4 showing a shift to the right. Again, a residual attended locus in the central region seems to be present.

Figure 7: Contour plot of RTs obtained in experiment 5. There seem to be two attended areas, one to the right of center, one to the left, but not as eccentrically located as in the previous two experiments.

\section{Discussion}

The present study indicates that in spite of the variability of RTs (much larger than the effects of eccentricity in any of the experimental conditions here investigated), some qualitative indication of the distribution of visual attention could be obtained with just ten subjects, each tested in a single session. Thus each point on the grid shown in Figure 1 was characterized by just ten RTs in each of the five experiments (Figs 3-7). The present results encourage increasing the sample size to obtain clearer images of attentional distributions, with higher spatial resolution and less noise. 
There is some indication that the center of the visual field may be somewhat attended at all times, even when subjects were instructed to pay attention to the right or to the left of center. When they were required to attend both to the right and to the left, they seemed to have some difficulty in separating the two foci. This was both evident from the lower eccentricity of the regions with fast RTs in experiment 5 when compared to experiments 3 and 4, and from residual fast RTs in the central area in both experiments 3 and 4. Still, at least qualitatively, Figs 3-7 have the broad characteristics that twould be expected from supposing that attention can be distributed to a specific area and also, to some extent, to two separate areas at the same time.

\section{References}

Awh, E., Pashler, H. (2000). Evidence for split attentional foci. Journal of Experimental Psychology: Human Perception and Performance, 26, No. 2, 834-846.

Downing, C. J., \& Pinker, S. (1985). The spatial structure of visual attention. In M. I. Posner \& O. S. M. Marin (Eds.). Attention and performance XI: Mechanisms of attention. Hillsdale, N. J.: Erlbaum.

Eimer, M. (1999). Attending to quadrants and ring-shaped regions: EPR effects of visual attention in different spatial selection tasks. Psychophysiology 56:42-52.

Eriksen, C. W., St. James, J. D. (1986). Visual attention within and around the field of focal attention: A zoom lens model. Perception \& Psychophysics, 40, 225-240.

Eriksen, C.W., Yeh, Y.Y. (1985). Allocation of attention in the visual field. Journal of Experimental Psychology: Human Perception and Performance, 11: 583-598

Gobell, J. L., Tseng, C.; Sperling, G. J. L. (2004) The spatial distribution of visual attention. Vision Research, 44, 1273-1296.

Heinze, H., Luck, S.J., Muente, T.F., Goes, A., Mangun, G.R. and Hillyard, S.A. (1994). Attention to adjacent and separate positions in space: An electrophysiological analysis. Perception and Psychophysics, 56:42-52.

LaBerge (1995). Attentional processing: the brain's art of mindfulness. Perspectives in cognitive neuroscience. Cambridge, MA: Harvard University Press.

Müller, M. M.; Malinowski, P.; Gruber, T.; Hillyard, S. A. (2003). Sustained division of the attentional spotlight. Nature, 424, 309-312.

Pan, K., \& Eriksen, C. (1993). Attentional distribution in the visual field during samedifferent judgments as assessed by response competition. Perception \& Psychophysics, 53, 134-144.

Posner, M. I. (1980). Orienting of attention. Quarterly Journal of Experimental Psychology, $32,3-25$.

Segalowitz, S. J. and Graves, R. (1990). Suitability of the IBM XT, AT and PS/2 keyboard, mouse, and game port as response devices in reaction time paradigms. Behavior Research Methods, Instruments, and Computers, 22, 283-289.

Schneider, W., Eschman, A., Zuccolotto, A (2002). E-Prime user's guide. Pittsburgh: Psychology Software Tools Inc. 
Proceedings of the XXth Annual Meeting of the International Society for Psychophysics Fechner' Day 2004

Acknowledgements. This research was supported by grants from CNPq, LHMCP has a CNPq doctoral fellowship 
Anexo 4

- Trabalho apresentado no "2005 ARVO Annual Meeting (Association for Research in Vision and Ophthalmology)" em Fort Lauderdale, EUA.

- Resumo publicado no periódico Invest. Ophthalmol. Vis. Sci., v. 46, E-Abstract $5653,2005$. 


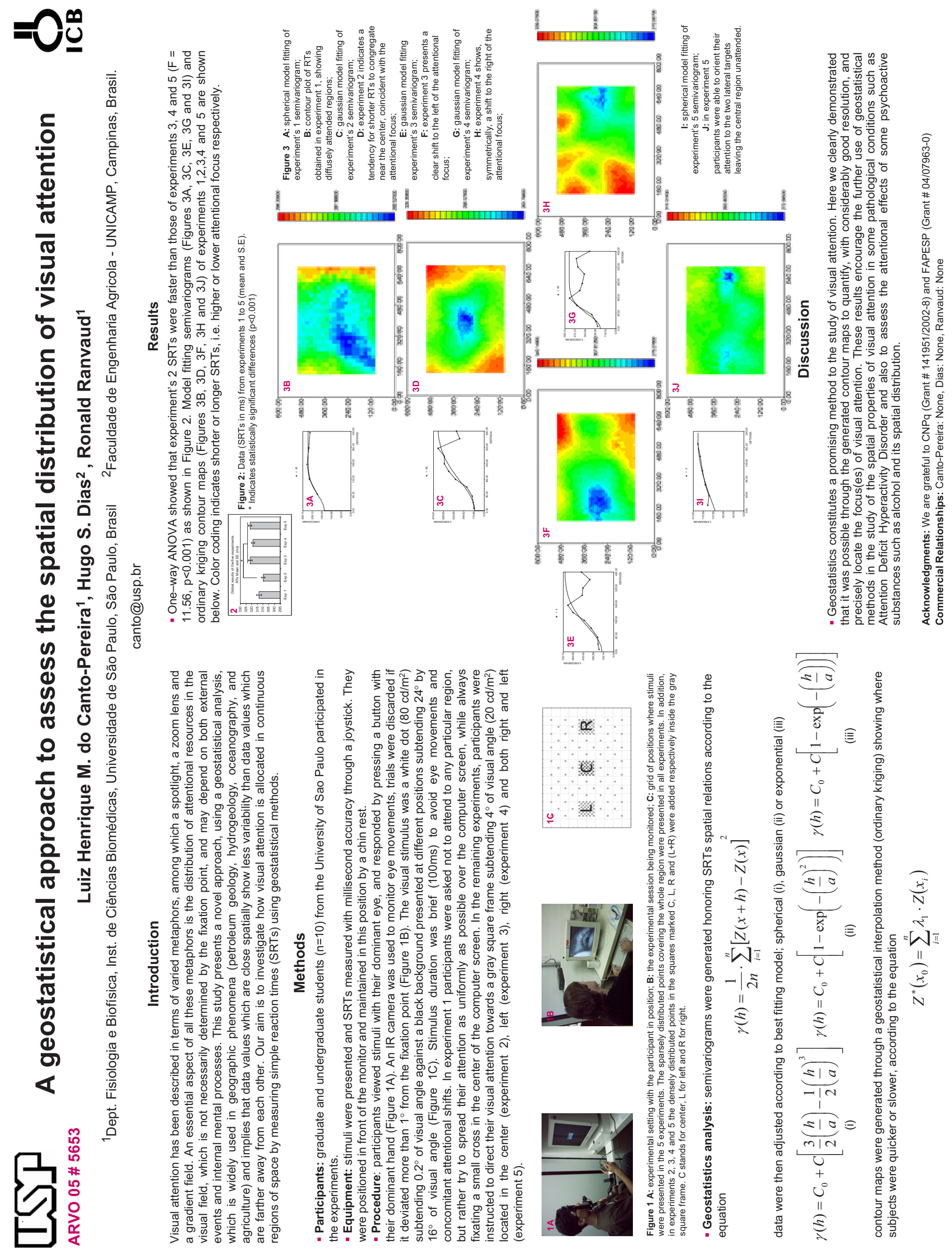




\section{Investigative Ophthalmology \& Visual Science}

SUBSCRIPTIONS

ARCHIVE SEARCH

Invest Ophthalmol Vis Sci 2005;46: E-Abstract 5653. (C) 2005 ARVO

$5653-B 856$

\section{A Geostatistical Approach to Assess the Spatial Distribution of Visual Attention}

\author{
L.H. M. Canto-Pereira ${ }^{1}$, R. Ranvaud ${ }^{1}$ and H.S. Dias $^{2}$ \\ ${ }^{1}$ Dept Physiology and Biophysics, Inst Biomed Sci Univ Sao Paulo, Sao \\ Paulo, Brazil \\ ${ }^{2}$ Fac. Engenharia Agricola - UNICAMP, Campinas, Brazil
}

QUICK SEARCH: [advanced]

\begin{tabular}{|c|c|c|c|}
\hline & Authc & & Keyword(s): \\
\hline Go & canto & \multirow[b]{2}{*}{ Vol: } & \\
\hline ear & & & Page: \\
\hline
\end{tabular}

Commercial Relationships: L.H.M. Canto-Pereira, None; R. Ranvaud, None; H.S. Dias, None.

Support: CNPq Grant \# 141951/2002-8

\begin{abstract}
Purpose: In the past twenty five years visual attention has been described in terms of varied metaphors, among which a spotlight, a zoom lens and a gradient field. An essential aspect of all these metaphors is the distribution of attentional resources in the visual field, which is not necessarily determined by the fixation point, and may depend on both external events and internal mental processes. This study presents a novel approach, using geostatistical analysis, to investigate how visual attention is allocated in continuous regions of space by measuring simple reaction times (SRTs) of subjects under different task requirements.
\end{abstract}

Methods: SRTs to visual stimuli were measured in ten young subjects (27.1 \pm 6.8 years). Stimuli and SRTs were provided by the software E-Prime v 1.1 (PST Inc.) using a 17' monitor driven by a Matrox P650 graphics board (refresh rate $100 \mathrm{~Hz}$, resolution of 800 x 600). Five different situations were employed: in experiment 1 participants were asked not to attend to any particular region, but rather try to spread their attention as uniformly as possible over the computer screen (diffuse attention). In the remaining experiments, participants were instructed to direct their visual attention covertly to the center (exp. 2), to the left (exp. 3), to the right (exp. 4) or to both right and left (but not to the center, thus characterizing a divided attention situation - exp. 5). Data were analyzed with the software Idrisi - Kilimanjaro v. 14.2 - Clark Labs - with Gstat geostatistical module.

Results: The isoline maps obtained through the geostatistical analysis show much structure, superposed on which there is a general tendency towards shorter times to the left in exp. 3, to the right in exp. 4, and distributed to both right and left (even though not as lateralized as in the previous two experiments) in exp. 5. One-way ANOVA showed that the RTs of experiment 2 were overall faster than those of experiments 3 , 4 and $5(\mathrm{~F}=11.56, \mathrm{p}<0.001)$. 
Anexo 5

- Trabalho apresentado no "Vision Sciences Society 2005 Annual Meeting".

- Resumo publicado no periódico Journal of Vision, 2005, v. 5, n. 8, p. 687. 


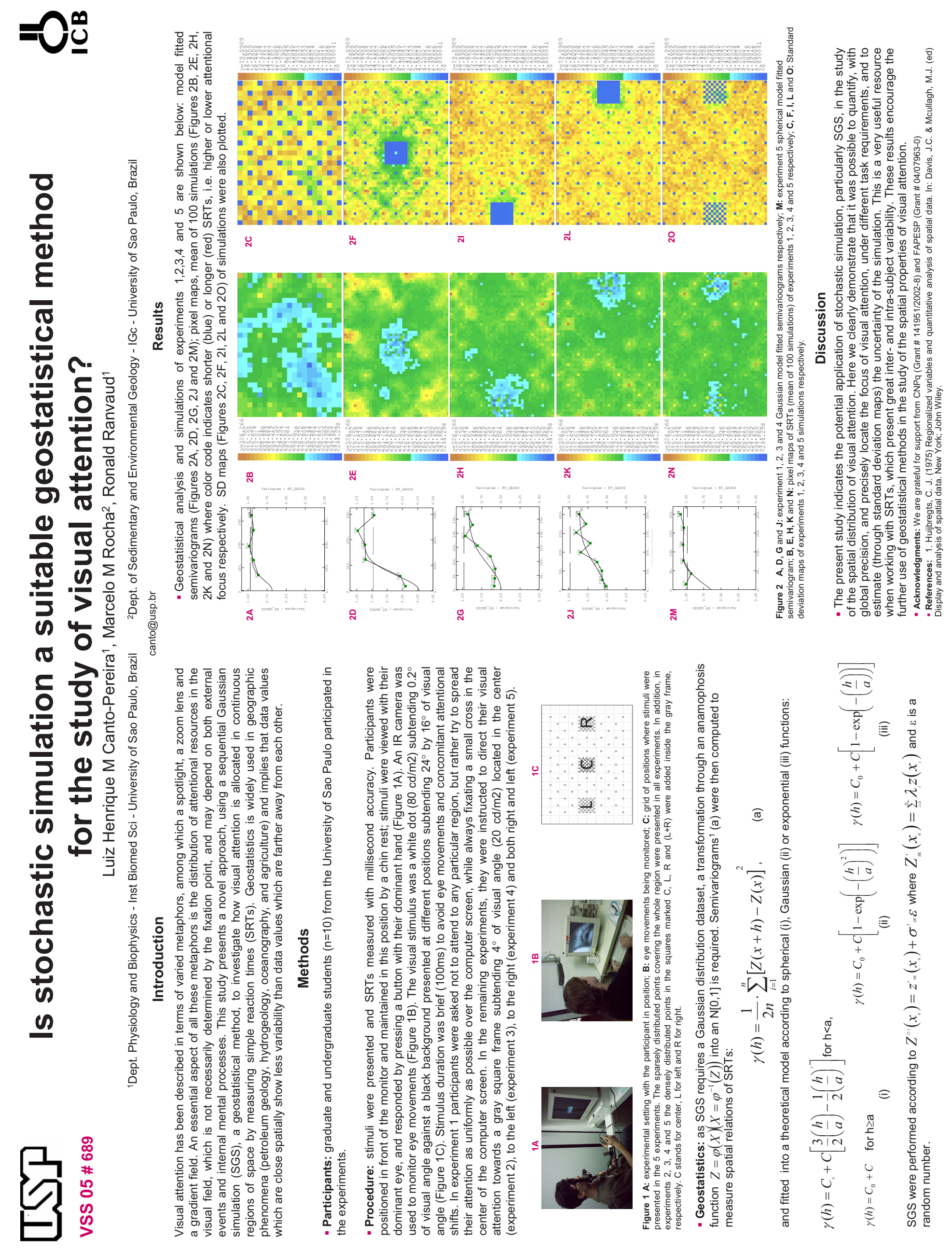




\section{VISION}

\section{Is Stochastic Simulation a Suitable Geostatistical Method for the Study of Visual Attention?}

\author{
Luiz Henrique M. \\ Canto-Pereira \\ Marcelo M. Rocha \\ Ronald Ranvaud
}

\author{
Dept. Physiology and Biophysics - Inst Biomed Sci - University of Sao \\ Paulo, Brazil \\ Dept. of Sedimentary and Environmental Geology - IGc - University of \\ Sao Paulo, Brazil \\ Dept. Physiology and Biophysics - Inst Biomed Sci - University of Sao
}

\section{Abstract}

Visual attention has been the subject of different metaphors including a spotlight (Posner, 1980), a zoom lens (Ericksen and St.James, 1986), and a gradient field (LaBerge, 1995; Downing and Pinker, 1985). This study proposes a novel paradigm to investigate the spatial distribution of visual attention. Simple reaction times (SRTs) to dots presented over the visual field were used to assess attentional allocation in space. We analyzed the data with several geostatistical methods. One of these, stochastic simulation, has been used in various fields, such as petroleum geology, hydrogeology, meteorology, and oceanography and seems to be particularly suitable for our purposes because it emphasizes spatial continuity patterns. Geostatistical stochastic simulation has the advantage of global precision, in other words it reproduces both the spatial variance and the statistical distribution characteristics of the phenomenon under study. As in any geostatistical method the basic tool is the variogram, which is used to predict data at any point within the domain. Simulations provide several different scenarios of equal probability, with the same spatial statistics of the original data. We used 5 different tasks and through SRTs we assessed attention (shorter or longer RTs were taken to indicate, respectively, higher or lower attentional focus). In experiment 1 participants were asked not to attend to any particular region, but rather try to spread their attention as uniformly as possible over the computer screen (diffuse attention). In the remaining experiments, subjects were instructed to direct their visual attention covertly to the center (experiment 2), to the left (experiment 3), to the right (experiment 4) or to both right and left, but not to the center, characterizing a divided attention situation (experiment $5)$.

We are grateful to CNPq (Grant \# 141951/2002-8)

\section{History}

Received September 15, 2005; published September 23, 2005

\section{Citation}

Canto-Pereira, L. M., Rocha, M. M., \& Ranvaud, R. (2005). Is Stochastic Simulation a Suitable Geostatistical Method for the Study of Visual Attention? [Abstract]. Journal of Vision, 5(8), 687a, http://journalofvision.org/5/8/687/, doi:10.1167/5.8.687.

\section{Keywords \\ None}

\section{On-Line Presentation}


Anexo 6

- Trabalho apresentado no "2006 ARVO Annual Meeting (Association for Research in Vision and Ophthalmology)" em Fort Lauderdale, EUA

- Resumo publicado no periódico Invest. Ophthalmol. Vis. Sci., v. 47, E-Abstract $3688,2006$. 


\section{Investigative Ophthalmology $\&$ Visual Science}

HOME HELP FEEDBACK
SUBSCRIPTIONS
ARCHIVE SEARCH
QUICK SEARCH:

[advanced]

\begin{tabular}{c|l|l} 
Author: & Keyword(s): \\
\hline Go $\mid$ pereira & \\
\hline Year: & Vol: & Page:
\end{tabular}

Invest Ophthalmol Vis Sci 2006;47: E-Abstract 3688. (C) 2006 ARVO

$3688-B 331$
Services

- Email this article to a friend

- Similar articles in this journal

- Alert me to new issues of the journal

- Download to citation manager

Google Scholar

\section{Anisotropic Distribution of Spatial Visual Attention}

\author{
L.H. Canto-Pereira and R.D. Ranvaud \\ Department of Physiology and Biophysics, University of Sao Paulo - \\ Institute of Biomedical Sciences, Sao Paulo, Brazil
}

Commercial Relationships: L.H. Canto-Pereira, None; R.D. Ranvaud, None.

Support: CNPq Grant \# 141951/2002-8 / FAPESP Grant \# 04/07963-0
Articles by Canto-Pereira, L.H.

- Articles by Ranvaud, R.D.

PubMed
Articles by Canto-Pereira, L.H.

Articles by Ranvaud, R.D.

\begin{abstract}
Purpose: We showed previously that geostatistical methods constitute a promising tool to assess the spatial distribution of visual attention (Canto-Pereira et al., ARVO 2005 \# 5653). In the present study, we investigated the anisotropic patterns of spatial visual attention under different tasks.

Methods: Reaction times (RTs) to visual stimuli were measured monocularly in 20 normal participants. Stimuli and RTs were provided by the software E-Prime v 1.1 (PST Inc.) using a 19" monitor driven by a 10 bit graphics board (refresh rate $100 \mathrm{~Hz}$, resolution of $800 \mathrm{x} 600$ ). Two different conditions were employed: in experiment 1 participants were asked not to attend to any particular region, but rather try to spread their attention as uniformly as possible over the computer screen (diffused attention) whereas in experiment 2 they were instructed to direct their attention toward the center of the computer screen coincident with the fixation point (overt attention).
\end{abstract}

Results: In experiment 1, 2D maps obtained through ordinary kriging (an interpolation method of geostatistical analysis) showed an attentional inferonasal advantage. Experiment 2 showed that participants indeed focused their attention toward the center of the screen, with spatial distribution similar to the topography of cone distribution of the human retina (Curcio et al. 1990).

Conclusions: An anisotropic pattern in the distribution of visuo-spatial attention was clearly evident under a diffuse attention situation; whereas in an overt attention situation, participants were able to focus their attention in a specific area of the computer screen.

Key Words: perception $\bullet$ visual fields $\bullet$ space and scene perception 
Anexo 7

- Trabalho apresentado no "Neuroscience 2006" em Atlanta, EUA 


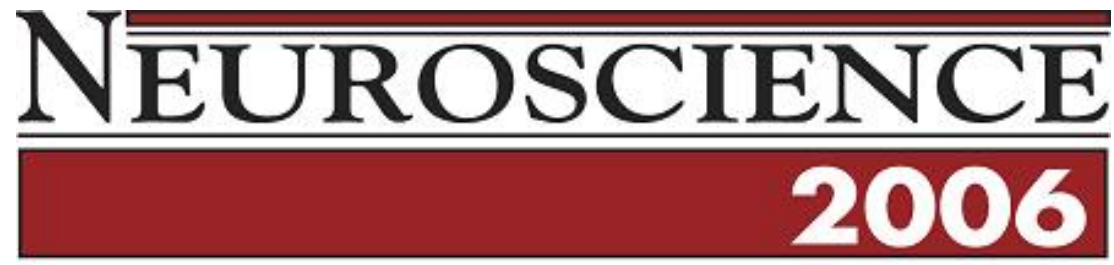

Print this Page for Your Records

Close Window

Control/Tracking Number: 2006-A-10892-SfN

Activity: Scientific Abstract

Current Date/Time: 5/15/2006 2:50:16 PM

Spatial distribution of visual attention under simple and choice reaction times paradigms.

Author Block: *L. M. Canto-Pereira ${ }^{1}$, F. Ferlazzo ${ }^{2}$, R. D. Ranvaud ${ }^{1}$;

${ }^{1}$ Physiology an Biophysics Department, University of Sao Paulo, Sao Paulo, BRAZIL, ${ }^{2}$ Dipartimento di Psicologia, Università di Roma "La Sapienza", Roma, ITALY.

In previous work we showed that visual attention is distributed preferentially in the lower visual hemifield under a diffuse attention situation: participants had no prior information as to where a target would appear in a simple reaction time task. Attention was evaluated through reaction times and geostatistical analysis of the data. Here we present choice reaction time data under the same diffuse attention situation. The rationale for this work was that in a simple reaction time task the location of the stimulus would have priority processing (dorsal visual pathway), which would be consistent with the lower visual hemifield advantage mentioned above. In a choice reaction time task, on the other hand, the nature of the stimulus, rather than its location, would require special resources, thus mobilizing the ventral visual pathway in order to correctly identify the stimulus, and respond appropriately (index finger press if the stimulus was a circle or middle finger press if the stimulus was a cross). If this reasoning is correct we would expect different spatial distributions of visual attention in these two situations. Indeed, 2D pixel plots obtained through geostatistical analysis of choice reaction times did not show better performance in the lower visual hemifield, but rather a larger roughly symmetrical parafoveal spread. The results so far confirm the general idea of dorsal/ventral visual pathway contribution in these experiments and their relation to the spatial distribution of visual attention in the two circumstances, characterized by "where" vs. "what". This encourages further experiments that segregate more precisely ventral and dorsal streams to better understand this issue.

Author Disclosure Block: L.M. Canto-Pereira, None; F. Ferlazzo, None; R.D. Ranvaud, None.

Theme and Topic (Complete): E.1.c. Attention ; C.4.1. Visual cognition: Attention and search

Keyword (Complete): ATTENTION ; VISUAL PERCEPTION ; VISION

Presentation Preference (Complete): \&nbspPoster Only

Support (Complete):

*Support: : Yes

Grant/Other Support: : CNPq Grant \# 141951/2002-8

Grant/Other Support: : FAPESP Grant \# 04/07963-0

Linking Group (Complete): None selected

Special Requests (Complete):

Religious Conflict? : No Religious Conflict

Status: Finalized 
Anexo 8

- Trabalho apresentado no "INA-10 - The 10th Meeting of the International Neurotoxicology Association, 2005” em Porvoo, Finlândia. 


\title{
The effect of alcohol on the spatial distribution of visual attention
}

uiz Henrique M. do Canto-Pereira ${ }^{1}$, Isabel A. David ${ }^{2}$, Walther Machado-Pinheiro² ${ }^{2}$ Ronald Ranvaud ${ }^{1}$

${ }^{1}$ Dept. Fisiologia e Biofísica, Instituto de Ciências Biomédicas I, Universidade de São Paulo, São Paulo, Brasil

${ }^{2}$ Centro de Ciências Médicas, Dept. de Fisiologia, Instituto Biomédico, Universidade Federal Fluminense, Brasil

\author{
canto@usp.br
}

\section{Introduction}

Visual attention has been described in terms of varied metaphors, among which a spotlight, a zoom lens and a gradient field. An essential aspect of all these metaphors is the distribution of attentional resources in the visual field, which is not necessarily determined by the fixation point, and may depend on both external events and internal mental processes. Alcohol intoxication is thought to disrupt the allocation of attention and impair divided attention situations. Also it has been proposed that alcohol has different effects on exogenous and endogenous attentional processes, the former being less affected than the later. This study presents a novel approach, based on geostatistical analysis, which is widely used in geographic phenomena in which data values are not independent, there being less variability in closely spaced points than in widely spaced points.

Our aim was to investigate how visual attention is affected by intoxication by alcohol in continuous regions of space by measuring simple reaction times (SRTs) over a large area of the visual field.

\section{Methods}

- Participants: graduate and undergraduate students $(n=24)$ from the University of Sao Paulo participated in the experiments, they were divided into two groups: control ( $n=10)$ and alcohol $(\mathrm{n}=14)$. Inclusion criteria were as follows: recent experience with the dose of alcohol to be administered, no history of medical condition that might contraindicate alcohol use, 20/25 Snellen best-corrected visual acuity or better and absence of known ophthalmological pathologies. The experiments were performed after all subjects had received adequate information on the experiment and had signed their informed consent.

- Equipment: stimuli were presented and SRTs measured with millisecond accuracy through a joystick. Participants were positioned in front of the monitor and maintained in this position by a chin rest.

- Procedure: participants viewed stimuli with their dominant eye, and responded by pressing a button with their dominant hand (Figure $1 \mathrm{~A})$. An IR camera was used to monitor eye movements, trials were discarded if it deviated more than $1^{\circ}$ from the fixation point (Figure 1B). The visual stimulus was a white dot $\left(80 \mathrm{~cd} / \mathrm{m}^{2}\right)$ subtending $0.2^{\circ}$ of visual angle against a black background presented at different positions in a region subtending $24^{\circ}$ by $16^{\circ}$ of visual angle (Figure $1 \mathrm{C}$ ). Stimulus duration was brief (100ms) to avoid eye movements and concomitant attentional shifts. In experiment 1 participants were asked not to attend to any particular region, but rather try to spread their attention as uniformly as possible over the computer screen since stimuli were equally probable everywhere. In the remaining experiments, participants were instructed to direct their visual attention towards a gray square frame subtending $4^{\circ}$ of visual angle $\left(20 \mathrm{~cd} / \mathrm{m}^{2}\right)$, where stimulus probability was higher than elsewhere, located in the center (experiment 2 ), to the left (experiment 3), to the right right (experiment 4) and both right and left (experiment 5). In the alcohol group, ethanol was administered in order to reach a blood alcohol concentration (BAC) of $0.06 \%$, therefore an ethanol dose of $0.4 \mathrm{~g} / \mathrm{Kg}$ was used through a drink made of vodka and orange juice; BACs were determined indirectly by means of a breathalyzer (Alcohawk ABI Digital Breath Alcohol Screener) and measurements were taken every 5 minutes.
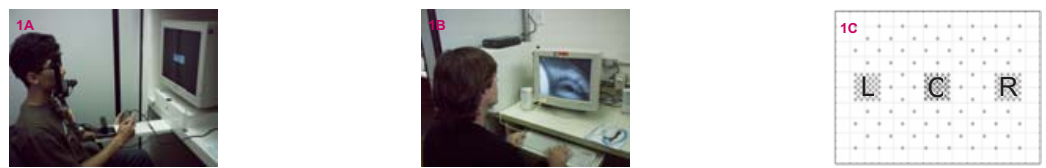

Figure 1 A: experimental setting with the participant in position; B: eye movements being monitored; C: grid of positions where stimuli were presented in the 5 experiments. The sparsely distributed points covering the whole region were presented in all experiments. In addition, in experiments $2,3,4$ and 5 the densely distributed points in the squares marked $C, L, R$ and $(L+R)$ were added inside the gray frame, respectively. $C$ stands for center, $L$ for left and $R$ for right.

- Geostatistics: semivariograms were generated honoring SRTs spatial relations according to the equation (i), data were then adjusted according to best fitting model. Contour maps were generated through an ordinary kriging (ii) which is a geostatistical interpolation method, showing where subjects were quicker or slower.
(i) $\gamma(h)=\frac{1}{2 n} \cdot \sum_{i=1}^{n}[Z(x+h)-Z(x)]^{2}$
(ii) $Z^{*}\left(x_{0}\right)=\sum_{i=1}^{n} \lambda_{1} \cdot Z\left(x_{i}\right)$

Results

- One-way repeated measures ANOVA showed that controls were faster than alcohol group in experiment 1 and alcohol group were faster in experiments 3 and 4 ( $F=5.44$, $\mathrm{p}<0.05$ ) as shown in Figure 2. Pixel maps obtained through ordinary kriging (Figures $3 \mathrm{~A}$ through $3 \mathrm{~J}$ ) of experiments $1,2,3,4$ and 5 are shown below. Color coding indicates shorter or longer SRTs, i.e. higher or lower attentional focus respectively. Figure 4 shows BACs measurements during the experimental setting.

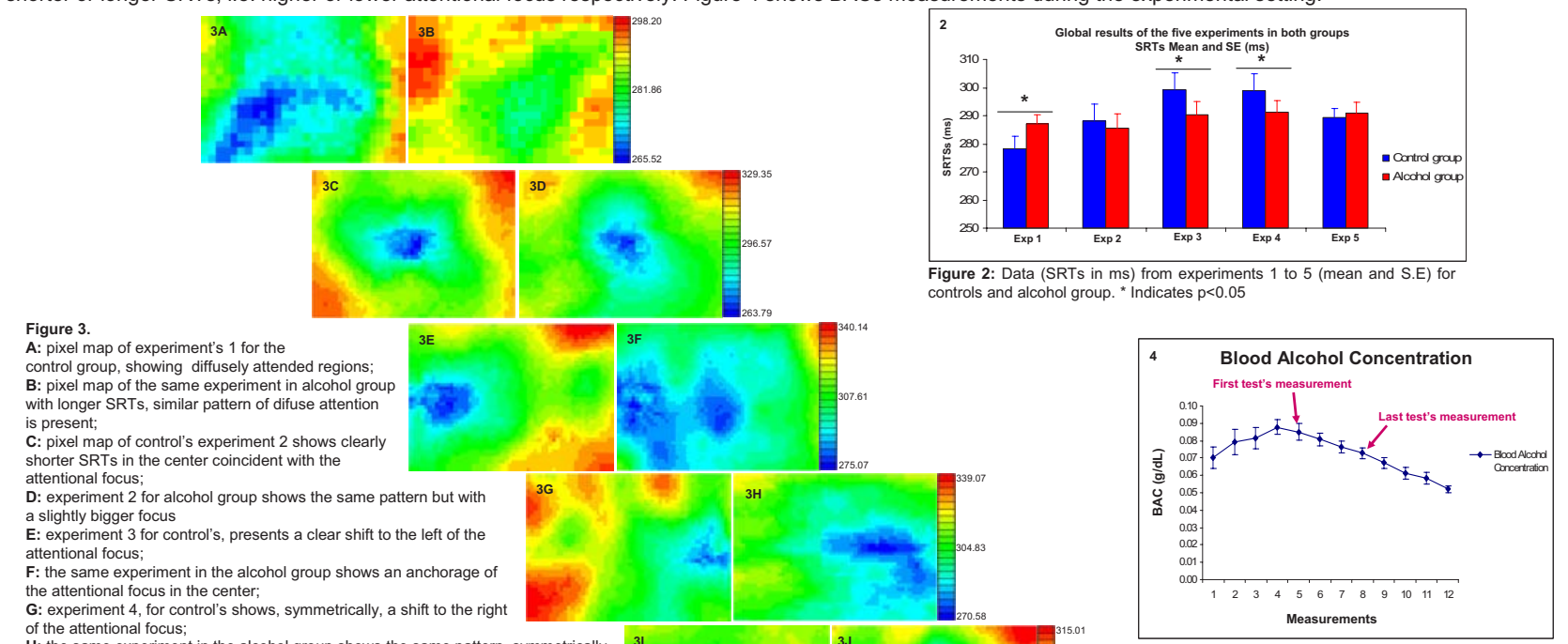

H: the same experiment in the alcohol group shows the same pattern, symmetrically, as in experiment 3 but with a shift to the right even though without disengaging from the center;

I: in experiment 5 controls were able to orient their attention to the two lateral targets leaving the central region unattended;

alcohol group, it wasn't possible to leave the central region unattended as for controls.

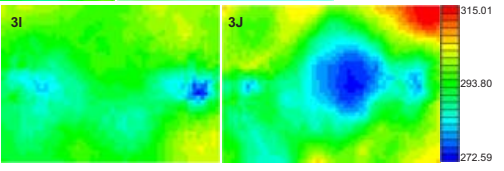

Figure 4: BACs (g/dL) measurements during the experimenta setting for the alcohol group in 5 minutes' intervals.

\section{Discussion}

- Alcohol intoxication impairs covert attention as shown in experiments' 3, 4 and 5 pixel maps.

- Alcohol specifically disrupt the ability to disengage attention from eye gaze, i.e. fixation point.

- Consonant to other sudies, alcohol intoxication impairs the ability to divide attention as shown in experiment 5.

- Here we clearly demonstrated that it was possible through the pixel maps to quantify, with good resolution, and precisely locate the focus(i) of visual attention and the effects of alcohol intoxication on its spatial distribution. 
Anexo 9

- Trabalho aceito para publicação no periódico Human \& Experimental Toxicology. 
Canto-Pereira et al. Alcohol and attention - Human \& Experimental Toxicology

\section{Effects of acute alcohol intoxication on visuo-spatial attention.}

Luiz Henrique M. do Canto-Pereira ${ }^{1}$, Isabel de P. A. David ${ }^{2}$, Walter Machado-Pinheiro ${ }^{3}$, Ronald D. Ranvaud ${ }^{1}$

${ }^{1}$ Department of Physiology and Biophysics, Institute of Biomedical Science and Neuroscience and Behavior Programs, University of São Paulo, São Paulo, Brazil.

${ }^{2}$ Department of Physiology, Biophysics Institute, Rio de Janeiro Federal University, Brazil.

${ }^{3}$ Department of Physiology and Pharmacology, Biomedical Institute, Federal Fluminense University, Brazil.

Address correspondence: Luiz Henrique M. do Canto-Pereira, Department of Physiology and Biophysics, Institute of Biomedical Sciences I, University of São Paulo, Av. Prof. Lineu Prestes, 1524, 05508-900 - São Paulo - SP - Brazil .E-mail: canto@usp.br 
Canto-Pereira et al. Alcohol and attention - Human \& Experimental Toxicology

The aim of the present study was to examine the effects of acute alcohol intoxication on the spatial distribution of visual attention measured with simple reaction times (RTs) to targets presented over an extended region of the visual field. Control $(n=10)$ and alcohol groups $(n=14)$ were tested with the same protocol. Participants were tested in 2 different conditions; in Experiment I, participants were instructed to direct their visual attention to the center, while in Experiment II they were asked to orient their attention covertly to both right and left, but not to the center. Throughout participants were required to fixate a small cross in the center of the computer screen. In the alcohol group, participants received an alcohol dose of $0.4 \mathrm{~g} / \mathrm{kg}$ so as to produce a Blood Alcohol Concentration (BAC) in the range of $0.08 \%$ during the experiments. The spatial distribution of reaction times was analyzed graphically with geostatistical methods and statistically through analysis of variance of particular regions of the visual field. Results showed that controls were able to direct their attention tightly towards the center (Expt I) and also to divide attention (Expt II) to the right and left. Participants in the alcohol group fixed their attention more diffusely in the center (Expt I) and were unable to disengage attention from the center in Experiment II. We conclude that acute alcohol intoxication impairs the ability to dissociate attention from gaze.

Keywords: visual attention, simple reaction times, spatial distribution, acute alcohol intoxication, geostatistics, divided attention. 
Canto-Pereira et al. Alcohol and attention - Human \& Experimental Toxicology

\section{Introduction}

The effects of alcohol on task performance have been extensively investigated. However, we do not known precisely all the mechanisms by which this drug exerts its effects. Although alcohol use, in general, is socially accepted, countless deleterious effects have been recognized. Acute alcohol intoxication is closely associated with car accidents, as well as antisocial acts and risk attitudes. ${ }^{1,2,3,4}$ The role of alcohol in such behaviors has been related with the negative effects of this drug on cognitive processes, especially on attention. ${ }^{5,6,7,8}$

In the past twenty years visual attention has been described in terms of varied metaphors, among which a spotlight ${ }^{9}$, a zoom lens ${ }^{10}$, and a gradient field. ${ }^{11,12}$ An essential aspect of all these metaphors is the distribution of attentional resources in the visual field, which is not necessarily determined by the fixation point, and may depend on both external events and internal mental processes.

A much-debated question is whether the flexibility of attentional distribution in space includes the possibility of attending to two separate areas, to the exclusion of the space between them. Awh and Pashler ${ }^{13}$, for example, present recent evidence in favor of the possibility of split attentional foci, whereas several prior Experiments seemed to question this possibility. ${ }^{14,15,16,17}$ Other evidence in favour of the two spatially separate attentional foci comes from Müller et al. ${ }^{18}$, who recorded evoked potentials in the visual primary cortex of human subjects, showing elegant contour maps of cortical activity as the subjects were exposed to different stimuli. Further recent research has proposed a more flexible view of this subject. For instance, Gobell et al. ${ }^{19}$ studied a task where subjects should spread their attention to multiple disjoint locations. In order to reinforce the division of attention, subjects were forced to ignore distracters positioned in the unattended locations; with this procedure subjects were 
Canto-Pereira et al. Alcohol and attention - Human \& Experimental Toxicology

able to promote a more efficient suppression of the intervening regions, obtaining a clearer distribution of attention to separate locations. Additionally, they found that this capacity of splitting attention to multiple locations could be influenced by many other factors such as spatial frequency and target eccentricity. Kraft et al. ${ }^{20}$ found further evidence in favour of this. According to these authors, spatial distribution of attention is also influenced by task difficulty and by where attentional targets are located over hemifields, so many factors determine how visual attention is distributed in space.

One aspect that is frequently emphasized is how alcohol could affect allocation of visual attention (see Koelega for review). ${ }^{21}$ Although it is well known that alcohol impairs the performance in tasks that require attention, the evidence indicates that alcohol does not impair uniformly all aspects involved in selective attention. For instance, Fillmore et al. ${ }^{22}$ dissociated the effects alcohol on controlled and automatic allocation of attention. They found that alcohol reduces the influence of controlled processes whereas increases the influence of automatic, non-voluntary processes. This view is supported by others, and there is general agreement that alcohol consumption impairs voluntary or cognitive control of attention. $23,24,25,26$

Alcohol seems to impair the ability to voluntarily allocate and use attention in a variety of tasks; however, such effects are more pronounced when dealing with competing demands, i.e. when subjects are asked to detect a stimulus while executing another task. For example, Moskowitz and Sharma ${ }^{27}$ assessed the effects of alcohol on peripheral vision. They measured the time required to detect a stimulus located in the periphery while, at the same time, a central task was being executed, which consisted in counting the blinks of a central light. They showed a performance decrement in intoxicated subjects only when the detection task was simultaneous with the counting task. No impairment was observed when the detection task was 
performed alone, without the central blink counting. Post et al. ${ }^{28}$ also theorized that alcohol might constrict the spatial distribution of attention, impairing intoxicated subjects to notice stimuli occurring in their peripheral field of vision. They measured the manual reaction times to the offset of stimuli positioned in distinct eccentricities. They found that the higher the eccentricity, the slower was the response, both in controls and in the intoxicated group. Moreover, intoxicated subjects showed greater increases in reaction times to the most peripheral stimuli. Thus, acute consumption of alcohol affects allocation of spatial attention, and particularly impairs responses to peripheral stimuli.

The assumptions above are in agreement with the alcohol myopia model. ${ }^{5,29}$ According to this model attentional capacity is limited during acute alcohol intoxication making it impossible to properly encode all relevant stimuli in the environment. Thus, the limited attentional resources are preferentially allocated to solve the primary task or to process the stimuli which are more immediate. Consequently, fewer resources will be available for secondary task or stimuli, which are not related with the central task, resulting in performance impairment. Consistently with this hypothesis much research has demonstrated that tasks requiring subjects to divide their attention across distinct spatial locations or to more than one task are severely impaired by acute alcohol intoxication. ${ }^{7,21,30}$ Moreover, intoxicated subjects seem to give priority to processing of primary or central tasks, even in a situation where the secondary task has emotional significance. ${ }^{31,32}$ All these findings contribute evidence in favour of the hypothesis that alcohol produces a narrowing of cognitive processing, leading to an increment in the sensitivity to direct cues and a decrement in the sensitivity to information outside the main focus. 
Canto-Pereira et al. Alcohol and attention - Human \& Experimental Toxicology

In order to apply these theoretical ideas to real situations, more research is needed. In particular, an important aspect that requires clarification is the way that alcohol generally affects the spatial distribution of attention. Particularly interesting is the critical situation of attentional division, where two targets must be simultaneously attended in distinct and separated positions. Although the effect of alcohol on behavior has been attributed to the restriction of the attentional focus, no adequate method has been used to properly measure this effect.

In this study we use a novel graphical approach to the question of attentional distribution, based on geostatistical analysis. ${ }^{33,34,35,36,37,38,39,40}$ This method has been widely used in geographic phenomena (e.g. petroleum geology, hydrogeology, oceanography, and agriculture) and is useful when data present spatial dependence, i.e. when data values which are close spatially show less variability than data values that are far away from each other.

Our aim here is to investigate how the spatial distribution of visual attention is affected by acute alcohol intoxication, measuring simple reaction times (RTs) to stimuli presented over a large portion of the visual field. 
Canto-Pereira et al. Alcohol and attention - Human \& Experimental Toxicology

\section{Material and Methods}

Human subjects, equipment and procedure

Graduate and undergraduate students $(n=24)$ from the University of São Paulo participated in this study. They were divided into two groups: control $(n=10)$ and alcohol $(n=14)$. All subjects in the alcohol group were previously screened on the basis of their drinking habits and medical history. Inclusion criteria: (i) 20/25 Snellen best-corrected visual acuity or better; (ii) absence of known ophthalmological pathologies, (iii) absence of any medical condition that might contraindicate alcohol use, (iv) right handed and (v) right eye dominance. Exclusion criteria: any history of alcohol problems or other drug dependency. The pattern of alcohol consumption was assessed through individual interviews and classified according to Cahalan and Cisin. ${ }^{41}$ Thus, only moderate social drinkers were tested. Also, each subject had the body mass index $\left[\mathrm{BMI}=\mathrm{Wt} / \mathrm{Ht}^{2}\right]$ calculated in order to exclude the overweight or extremely thin individuals (Kuczmarski and Flegal). ${ }^{42}$ All the procedures were approved by the local ethics committee and, before the experiment, subjects sign the informed consent.

RTs were measured with the software E-Prime v 1.1 (PST Inc.) ${ }^{43}$ using a 19' Samsung 997 DF monitor powered by a PC Athlon XP 2400/512 driven by a 10-bit Matrox P650 graphics board with a refresh rate of $100 \mathrm{~Hz}$ and a resolution of $800 \mathrm{x}$ 600. Participants' responses (button presses) were registered with millisecond accuracy ${ }^{44}$ through a joystick connected to the game port of the PC. Participants were positioned in front of the monitor and maintained in this position during the experiment with their heads stabilized by a chin rest. An infrared camera was used to monitor eye movements during the experiments, and trials were discarded if the eye deviated more than $1^{\circ}$ from the fixation point. 
Canto-Pereira et al. Alcohol and attention - Human \& Experimental Toxicology

All experimental sessions were performed in a darkened, sound attenuated room. Participants viewed stimuli with their dominant right eye, and were requested to respond as quickly as possible to the onset of the target by pressing a button on the joystick with the index finger of the dominant right hand. Prior to data collection, subjects went through a training session allowing them to become acquainted with the procedure. Subjects were required to keep their gaze on a small cross in the center of the computer screen. The target to which subjects had to respond as quickly as possible was a white dot, subtending $0.2^{\circ}$ of visual angle, with a luminance of 80 $\mathrm{cd} / \mathrm{m}^{2}$, against a black background presented at different positions on the computer screen. Stimulus duration was brief $(100 \mathrm{~ms})$ to avoid eye movements and concomitant attentional shifts. ${ }^{44}$ RTs below $150 \mathrm{~ms}$ and above $500 \mathrm{~ms}$ were discarded.

In Experiment I participants were instructed to direct their visual attention towards a gray square frame subtending $4^{\circ}$ of visual angle, containing 80 target positions, and centered at the fixation cross; the rest of the screen had 78 target positions (Fig. 1A).

In Experiment II participants were instructed to keep their gaze in the fixation cross in the center of the screen but to direct their attention simultaneously towards two gray square frames subtending $4^{\circ}$ of visual angle, each one containing 41 target positions, , centered $10^{\circ}$ to the right and left of the fixation cross; the rest of the screen had 72 target positions (Fig. 1B).

Experiments were carried out in a counterbalanced way, i.e. half of the participants in both groups were tested first in Experiment I and then in Experiment II, while the other half of participants were tested in the reverse order.

Figure 1 shows the positions where the stimuli were presented in the two experiments. The grid of stimuli subtended $24^{\circ}$ by $16^{\circ}$ of visual angle, and each point 
Canto-Pereira et al. Alcohol and attention - Human \& Experimental Toxicology

in the grid was presented only once in each experiment, thus Experiment I consisted of 158 trials and Experiment II consisted of 154 trials. The inter-stimulus interval was randomly assigned between 750 and $1500 \mathrm{~ms}$.

Figure 1A here

Figure $1 B$ here

All procedures, except alcohol administration were the same for the control and for the alcohol group. In the alcohol group, participants were required not to drink ethanol for 24 hours and abstained from food for 2 hours prior to the testing. They were informed about the amount of alcohol to be administrated and the expected symptoms. Before beginning of the experimental session, each participant drank a mixture of vodka (Stolichnaya ${ }^{\circledR}$ ) containing $40 \%$ alcohol by volume and orange juice in a 1:1 ratio. The amount of alcohol to be consumed by each subject to reach a peak blood alcohol concentration (BAC) of approximately $0.08 \%$ during the test phase was calculated using a formula based upon the subject's weight $(0.4 \mathrm{~g}$ of ethanol per $\mathrm{kg}$ of body weight). Participants were instructed to drink the beverage within a period of 5 min and had to wait, after that, 25 minutes before data collection.

Blood alcohol concentration was measured only in the alcohol group and the BAC values were obtained indirectly by means of a breath alcohol analyzer (CA $2000^{\circledR}$ ). The first measurement occurred 10 minutes after the subjects had finished drinking. Following that first measurement a total of eleven further readings were 
Canto-Pereira et al. Alcohol and attention - Human \& Experimental Toxicology

taken at 5 min intervals. Thus, this procedure resulted in twelve alcohol concentration values (numbered from 1 to 12 covering the period from 10 to 65 minutes, after terminating alcohol ingestion). Thus, some measurements were acquired before (pretest phase), during (test phase) and after (post-test phase) the end of the acquisition of the experimental data. The critical measurements of the "test phase" were those obtained immediately before the beginning of data collection. Once the tests were finished, participants left the laboratory only after their BAC was below $0.03 \%$.

\section{Data Analysis}

Data were analyzed with standard commercial statistical software (SigmaStat 1.0 and Statistica 6.0).

A two-way repeated measures ANOVA was used to verify global differences between groups and experiments.

In order to verify if the qualitative difference in the geostatistical maps correspond to significant differences in RTs, we decide to run another ANOVA. The aim of this second ANOVA was to verify whether RTs of the central and lateral regions of interest (ROIs, i.e. the $4^{\circ} \times 4^{\circ}$ squares defined by gray frames, see Fig. 1) were different, and how they were influenced by the experimental conditions (Expts 1 and 2) for both experimental groups (control and alcohol). In these analyses, we calculated the mean RTs obtained for each subject in the central ROI and those obtained in the lateral ROIs. It is important to mention that RTs of both lateral (left and right) ROIs were pooled together in this analysis. Therefore, in this ANOVA, group (control and alcohol) was used as a between-subject factor, and instructions (pay attention to the center - Expt. 1, and pay attention toward the laterals - Expt. 2) and ROIs (central and laterals) were used as within-subject factors. Moreover, 
Canto-Pereira et al. Alcohol and attention - Human \& Experimental Toxicology

planned comparisons were done, within each group, to check the effects of experimental condition on RTs for both central and laterals ROIs.

RTs were also analyzed through a geostatistical method using GeoVisual software version 2.2. ${ }^{35}$ Geostatisitics is useful whenever data show spatial dependence, i.e. whenever data values which are close spatially show less variability than data values which are farther away from each other. The exact nature of this pattern varies from experiment to experiment. The variability of the data as a function of distance between points is a function called semivariogram, $\gamma(\mathrm{h})$, given by:

$$
\gamma(h)=\frac{1}{2 n} \cdot \sum_{i=1}^{n}[Z(x+h)-Z(x)]^{2},
$$

where $\mathrm{n}$ is the number of pairs of points separated by a distance $\mathrm{h}$; $\mathrm{Z}(\mathrm{x})$ is the value of the regionalized variable (data value) at the point $x ; Z(x+h)$ is the value the value of the regionalized variable at the point $(x+h)$. A detailed description of the geostatisitical analysis is given in Goovaerts. ${ }^{34}$

A unifactorial (12 levels) analysis of variance (ANOVA) was used to analyze variations on blood alcohol concentration, only in the alcohol group as a function of time following alcohol administration. Thus, BAC measurements (moments from 1 to 12) were used as within-subjects factor. When necessary, we also performed a posthoc analysis using the Newman-Keuls method. The significance level adopted was $\mathrm{p}<0.05$. 
Canto-Pereira et al. Alcohol and attention - Human \& Experimental Toxicology

\section{Results}

Error rate

Trials that were exclude $(\mathrm{RT}<150 \mathrm{~ms}$ or $>500 \mathrm{~ms}$, or trials were eye movements occurred) constituted less than $4 \%$ of the total number of trials in both experimental conditions and for both alcohol and control groups.

\section{Blood Alcohol Concentration}

Analysis of variance of BAC values measured at 12 moments following alcohol administration showed a statistically significant difference as would be expected during the hour following alcohol administration $\left[\mathrm{F}_{(11,143)}=12.41 ; \mathrm{p}<0.01\right]$. However, the post-hoc analysis showed that the mean BACs obtained during the time participants performed Experiments I and II (moments 4 to 7) did not differ from each other ( $p>0.05$ for all). Differences were only obtained when BACs of the test phase were compared to BACs obtained for the pre-test and/or post-test phase. Thus, the BACs values during the attentional tests did not vary significantly, presenting a mean value of $0.08 \pm 0.01 \%$.

$R T S$

Two-way repeated measures ANOVA showed that globally controls did not differ in their overall RTs from the alcohol group $\left[\mathrm{F}_{(1,22)}=0.016 ; \mathrm{p}=0.897\right]$, nor was any difference present between Experiments I and II $\left[\mathrm{F}_{(1,1)}=0.048 ; \mathrm{p}=0.827\right]$. These global results are shown in Figure 2.

Figure 2 here 
Canto-Pereira et al. Alcohol and attention - Human \& Experimental Toxicology

The second ANOVA, however, showed that ROIs $\left[\mathrm{F}_{(1,22)}=18.73 ; \mathrm{p}<0.001\right]$ was a significant source of variance, and also the interactions between instructions and ROIs $\left[\mathrm{F}_{(1,22)}=10.29 ; \mathrm{p}=0.004\right]$, and the triple interaction among group, instructions and ROIs $\left[\mathrm{F}_{(1,22)}=5.03 ; \mathrm{p}=0.03\right]$. In the factor ROIs, RTs for central stimuli were shorter $(278 \pm 31 \mathrm{~ms})$ than those obtained for lateral ones $(295 \pm 26 \mathrm{~ms})$. The triple interaction means that the effects of the instructions and ROIs between the two groups were different. Figure 3 shows the main results of this ANOVA. The planned comparisons confirm and extended the ANOVA results showing that: i) Control group - RTs obtained for the lateral stimuli were significantly shorter $(p=0.009)$ when subjects were instructed to pay attention toward the lateral ROIs $(283 \pm 24 \mathrm{~ms})$, in relation to the instruction to pay attention to the center $(310 \pm 35 \mathrm{~ms})$. For central stimuli, the difference between the instruction to pay attention to center $(271 \pm 20 \mathrm{~ms})$ and that to pay attention toward the sides $(302 \pm 46 \mathrm{~ms})$ also was marginally significant $(\mathrm{p}=0.053)$. Therefore, for the control groups, it is clear that the instructions of Expts I and II caused differences in RTs in both ROIs (Figure 3A). ii) Alcohol group - RTs obtained for the central stimuli were not affect by the instructions of Expts I and II: RTs obtained when the instruction was to pay attention to the center $(273 \pm 26 \mathrm{~ms})$, did not differ from those when the instruction was to pay attention toward the sides $(268 \pm 31$ $\mathrm{ms}, \mathrm{p}=0.692$ ). This means that, for this group, independently of the instructions, attention was always strong in the center. For lateral stimuli there was also marginal significance $(\mathrm{p}=0.061)$ for shorter RTs when subjects were instructed to pay attention toward the lateral ROIs $(286 \pm 18 \mathrm{~ms})$, in relation to the instruction to pay attention to the center $(302 \pm 45 \mathrm{~ms})$ (Figure 3B). Taken together, the data obtained for the alcohol group suggest that, despite the instructions they received, they always maintained their attention around the point of gaze. 
Canto-Pereira et al. Alcohol and attention - Human \& Experimental Toxicology

Figure $3 A$ here

Figure $3 B$ here

The data were also submitted to graphical analysis using geostatistical methods and then plotted as a pixel maps. Maps of Experiment I were not very different for the alcohol and the control groups, except that the attentional focus of the alcohol group appeared to be more diffuse that of controls. Maps of Experiment II however, showed a remarkable difference between the two groups. Whereas controls were able to split their attentional focus to both lateral targets (leaving the center relatively unattended), characterizing a divided attention situation; the alcohol group was not able to do so: their main attentional focus was coincident with the fixation point at all times. Figures 4A through 4D show the pixel maps obtained through ordinary kriging of Experiments I and II in both groups. Grayscale coding indicates shorter RTs as lighter areas and longer RTs as darker areas, indicating higher or lower attentional focus respectively.

Figure $4 A$ here 
Canto-Pereira et al. Alcohol and attention - Human \& Experimental Toxicology

Figure $4 B$ here

Figure $4 C$ here

Figure 4D here

\section{Discussion}

Acute alcohol intoxication disrupts the distribution of spatial attention, as shown in the pixel map of Experiment II (Fig 4D). Participants in the alcohol group maintained their attention tightly focused near the point of gaze, and thus presented impairment in endogenously preferentially orienting attention to peripheral regions, as shown not only by the pixel maps but also by the second ANOVA.

This tendency of intoxicated subjects to allocate their attention always around their point of gaze can be related to other findings. For example, Wegner and Fahle ${ }^{45}$ have demonstrated that alcohol intoxicated subjects take more advantage of a gap condition than sober volunteers in saccadic responses, i.e. they show an increased gap effect. The gap effect is the reduction observed in saccadic reaction times when $200 \mathrm{~ms}$ before the onset of a peripheral target, the fixation point disappears (gap 
Canto-Pereira et al. Alcohol and attention - Human \& Experimental Toxicology

condition), relative to the condition with no fixation point offset (overlap condition). $46,47,48$ Some authors interpret this latency reduction as a consequence of the disengagement of attention induced by the fixation point offset. ${ }^{47,49,50}$

According to this view, while attention is disengaged, the detection of any other stimuli will be facilitated and thus the saccadic response time would decrease. Wegner and Fahle ${ }^{45}$ showed that the amount of reduction in saccadic response time obtained in the gap condition for controls was $8 \%$ of the latency in the classic condition. On the other hand, the gain obtained under the influence of alcohol reached $18 \%$. This finding is consistent with the supposition that the sensitivity of intoxicated subjects to foveal stimuli is increased and that they tend to allocate attentional resources near the fovea.

An alternative explanation is based on the alcohol myopia model ${ }^{5}$, as mentioned in the introduction. According to this model, attentional capacity is reduced in intoxicated subjects, making it difficult to properly process all the relevant stimuli in the environment. This assumption gives rise to two possible explanations for the effects of alcohol on attentional tasks. In complex situations, especially when the primary task presents a high level of difficulty, inebriated subjects will allocate their limited attentional resources in the most important stimulus or the primary task. As result, the processing of secondary tasks or the effect of distracters will be greatly impaired. On the other hand, when the primary task is simple and low-demanding, more resources will be available for processing distracters and its disruptive effect on the primary task will become more evident. In our experiments, subjects did not execute a high demanding task. Thus, their primary task in Experiment II was to covertly orient their attention to the sides, in order to facilitate the visual perception of the stimulus that would probably appear in the attended location. Although sobers 
Canto-Pereira et al. Alcohol and attention - Human \& Experimental Toxicology

participants did not have any problems with this task, intoxicated participants had difficulty in properly orienting their attention to the indicated location shifting away from the fixation point. The effects of alcohol on the spatial spread of visual attention were clearly observed in the pixel map of Experiment II and in the second ANOVA. According to our interpretation, owing to the low-demanding characteristic of the primary task in our experiments, intoxicated participants were not able to ignore the "distractor stimulus" - the fixation point, presented in the fovea. Thus, alcohol would impair performance on attention by compromising the ability to direct attention to relevant positions (the targets) and away from the irrelevant one (the fixation point).

According to Kraft et al ${ }^{20}$, targets positioned in the same hemifield impair splitting of attention. Thus, it is easier to split attention across targets in opposite the hemifields than in the same hemifield. In agreement with this supposition, in our paradigm the stimuli were positioned in different hemifields and in the control situation we found evidence in favour of divided attention. ${ }^{51}$ In addition, it is also postulated by Kraft et al. ${ }^{20}$ that divided attention is more likely to occur in a task with high demanding characteristic.

In summary, our study showed that pixel maps obtained through geostatistical analysis are useful to show, with good resolution, and to precisely locate the focus(i) of visual attention, and that $\mathrm{t}$ alcohol intoxication affects the capacity to voluntarily allocate visual attention in space. These effects were evident in Experiment II where participants were required to divide their attention between two spatial locations before target appearance. In this condition, controls were able to split their attentional focus according to the instructions and responded faster to stimuli appearing in both locations. Intoxicated participants, on the other hand, presented impairment in this situation, focusing their attention primarily on the fixation point, i.e. they did not seem 
Canto-Pereira et al. Alcohol and attention - Human \& Experimental Toxicology

able to disengage their attention form their gaze. Although alcohol-related performance decrements are not restricted to only this situation, these results are compatible with those of others researchers who showed that alcohol is related to impairment in dividing attention. ${ }^{7,21,42}$

Geostatistical methods constitute a useful tool to analyze the effects of acute alcohol intoxication on the spatial distribution of visual attention. Through this method it was possible to visualize alcohol's effects on attention with a new perspective through pixel maps. It was possible to demonstrate that alcohol impaired disengagement of the focus of visual attention from gaze. Thus it appears that alcohol promotes an "attentional anchorage" to the location of the eye gaze.

In conclusion, in the present study we were able to demonstrate an attentional effect of acute alcohol intoxication. Geostatistics thus constitutes a promising resource to investigate attentional effects of other neurotoxicants and psychotropic drugs.

\section{Acknowledgements}

This research was supported by CNPq Grant \# 141951/2002-8 to LHMCP (doctoral fellowship). The first author is grateful to the local committee of the International Neurotoxicology Association (INA) for the financial support enabling him to present these results at the INA-10 meeting in Porvoo, Finland. We thank the anonymous reviewers for very helpful comments on a previous version of the article. 
Canto-Pereira et al. Alcohol and attention - Human \& Experimental Toxicology

\section{References}

1 Híjar M, Flores M, López MV, Rosovsky H. Alcohol intake and severity of injuries on highways in Mexico: a comparative analysis. Addiction 1998; 93: 1543-1551.

2 Giancola PR, Zeichner A, Yarnell JE, Dickson KE. Relation between executive cognitive functioning and the adverse consequences of alcohol use in social drinkers. Alcoholism: Clinical and Experimental Research 1996; 20: 1094-1098.

3 Cohen DA, Mason K, Scribner R. The population consumption model, alcohol control practices and alcohol-related traffic fatalities. Preventive Medicine 2001; 34: 187-197.

4 Farquhar K, Lambert K, Drummond GB, Tiplady B, Wright P. Effect of ethanol on psychomotor performance and on risk taking behaviour. Journal of Psychopharmacology 2002; 16: 379-384.

5 Steele CM, Josephs RA. Alcohol myopia: its prized and dangerous effects. American Psychologist 1990; 45: 921-933.

6 Herzog TA. Effects of alcohol intoxication on social inferences. Experimental and Clinical Psychopharmacology 1999; 7: 448-453.

7 Rosselló J, Munar E, Justo S, Arias R. Effects of alcohol on divided attention and on accuracy of attentional shift. Psychology in Spain 1999; 3: 69-74.

8 Bartholow BD, Pearson M, Sher KJ, Wieman LC, Fabiani M, Gratton G. Effects of alcohol consumption and alcohol susceptibility on cognition: a psychophysiological examination. Biological Psychology 2003; 64: 167-190.

9 Posner, MI. Orienting of attention. Quarterly Journal of Experimental Psychology 1980; 32: 3-25.

10 Eriksen CW, St. James JD. Visual attention within and around the field of focal attention: A zoom lens model. Perception \& Psychophysics 1986; 40: 225-240. 
Canto-Pereira et al. Alcohol and attention - Human \& Experimental Toxicology

11 Downing CJ, Pinker S. The spatial structure of visual attention. In M. I. Posner \& O. S. M. Marin (Eds.). Attention and performance XI: Mechanisms of attention. Hillsdale, N. J.: Erlbaum, 1985.

12 LaBerge D. Attentional processing: the brain's art of mindfulness. Perspectives in cognitive neuroscience. Cambridge, MA: Harvard University Press, 1995.

13 Awh E, Pashler H. Evidence for split attentional foci. Journal of Experimental Psychology: Human Perception and Performance, 2000; 26: 834-846.

14 Eriksen CW, Yeh YY. Allocation of attention in the visual field. Journal of Experimental Psychology: Human Perception and Performance 1985; 11: 583-598.

15 Pan, K., \& Eriksen, C. Attentional distribution in the visual field during samedifferent judgments as assessed by response competition. Perception \& Psychophysics 1993; 53: 134-144.

16 Heinze H, Luck SJ, Muente TF, Goes A, Mangun GR, Hillyard SA. Attention to adjacent and separate positions in space: An electrophysiological analysis. Perception and Psychophysics 1994; 56: 42-52.

17 Eimer M. Attending to quadrants and ring-shaped regions: EPR effects of visual attention in different spatial selection tasks. Psychophysiology 1999; 36: 491-503.

18 Müller M M, Malinowski P, Gruber T, Hillyard SA. Sustained division of the attentional spotlight. Nature 2003; 424: 309-312.

19 Gobell JL, Chia-huei T, Sperling G. The spatial distribution of visual attention. Vision Research 2004; 44:1273-1296.

20 Kraft A, Müller NG, Hagendorf H, Schira MM, Dick S, Fendrich RM, Brandt SA. Interactions between task difficulty and hemispheric distribution of attended locations: implications for the splitting attention debate. Cognitive Brain Research $2005 ; 24: 19-32$. 
Canto-Pereira et al. Alcohol and attention - Human \& Experimental Toxicology

21 Koelega HS. Alcohol and vigilance performance: a review. Psychopharmacology 1995; 118: 233-249.

22 Fillmore MT, Vogel-Sprott M, Gavrilescu D. Alcohol effects on intentional behavior: dissociating controlled and automatic influences. Experimental and Clinical Psychopharmacology 1999; 7: 372-378.

23 Peterson JB, Rothfleish J, Zelazo PD, Pihl RO. Acute alcohol intoxication and cognitive functioning. Journal of Studies on Alcohol 1990; 51: 114-122.

24 Giancola PR. Executive functioning: a conceptual framework for alcohol related aggression. Experimental and Clinical Psychopharmacology 2000; 8: 576-597.

25 Casbon TS, John JC, Lang AR, Patrick CJ. Deleterious effects of alcohol intoxication: diminished cognitive control and its behavioral consequences. Journal of Abnormal Psychology 2003; 112: 476-487.

26 Curtin JJ, Fairchild BA. Alcohol and cognitive control: implications for regulation of Behavior during response Conflict. Journal of Abnormal Psychology 2003; 112 : 424-436.

27 Moskowitz H, Sharma S. Effects of alcohol on peripheral vision as a function of attention. Human Factor 1974; 16: 174-180.

28 Post RB, Lott LA, Maddock RJ, Beede JI. An effect of alcohol on the distribution of spatial attention. Journal of Studies on Alcohol 1996; 57: 260-266.

29 Josephs RA, Steele CM. The two faces of alcohol myopia: attentional mediation of psychological stress. Journal of Abnormal Psychology 1990; 99: 115-126.

30 Schulte T, Müller-Oehring EM, Strasburger H, Warzel H, Sabel BA. Acute effects of alcohol on divided and covert attention in men. Psychopharmacology 2001; 154: 61-69. 
Canto-Pereira et al. Alcohol and attention - Human \& Experimental Toxicology

31 Curtin JJ, Lang AR, Patrick CJ, Strizke WGK. Alcohol and fear-potentiated startle: the role of competing cognitive demands in the stress-reducing effects of intoxication. Journal of Abnormal Psychology 1998; 107: 547-557.

32 Curtin JJ, Patrick CJ, Lang AR, Cacioppo JT, Birbaumer, N. Alcohol affects emotion through cognition. Psychological Science 2001; 12: 527-531.

33 Journel AG. Geostatistics: models and tools for the earth sciences. Mathematical Geology 1986; 18: 119-140.

34 Goovaerts P. Geostatistics for natural resources evaluation. Oxford University Press, New York, 1997.

35 Yamamoto JK. An Alternative measure of the reliability of ordinary kriging estimates. Mathematical Geology 2000; 32: 489-509.

36 Fuchs K, Deutz A. Use of variograms to detect critical spatial distances for the Knox's test. Preventive Veterinary Medicine 2002; 54: 37-45.

37 Ludovisia A, Minozzo M, Pandolfia P, Taticchia MI. Modelling the horizontal spatial structure of planktonic community in Lake Trasimeno (Umbria, Italy) using multivariate geostatistical methods. Ecological Modelling 2005; 181, 247-262.

38 Miranda ML, Dolinoy DC. Using GIS-Based Approaches to Support Research on Neurotoxicants and Other Children's Environmental Health Threats. Neurotoxicology 2005; 26: 223-228.

39 Mather FJ, White LE, Langlois EC, Shorter CF, Swalm CM, Shaffer JG, Hartley, WR. Satistical Methods for linking health, exposure, and hazards. Environmental Health Perspectives 2004; 112(14): 1440-1445.

40 Canto-Pereira LHM, Dias H, Ranvaud R. A geostatistical approach to assess the spatial distribution of visual attention. Investigative Ophthalmology and Visual Sicences 2005; 46: 5653. 
Canto-Pereira et al. Alcohol and attention - Human \& Experimental Toxicology

41 Cahalan D, Cisin IH. American drinking practices: Summary of findings from a national probability sample: I. Extent of drinking by population subgroup. Quarterly Journal of Studies on Alcohol 1968; 29: 130-151.

42 Kuczmarski RJ, Flegal KM. Criteria for definition of overweight in transition: background and recommendations for the United States. American Journal of Clinical Nutrition 2000; 72: 1074-1081.

43 Schneider W, Eschman A, Zuccolotto A. E-Prime user's guide. Pittsburgh: Psychology Software Tools Inc, 2002.

44 Segalowitz SJ, Graves R. Suitability of the IBM XT, AT and PS/2 keyboard, mouse, and game port as response devices in reaction time paradigms. Behavior Research Methods, Instruments, and Computers 1990; 22: 283-289.

44 Müller, M.M., Malinowski, P., Gruber, T \& Hillyard, S.A. Sustained division of the attentional spotlight. Nature 2003; 424: 309-312.

45 Wegner AJ, Fahle M. Alcohol and visually guided saccades: gap effect and predictability of target location. Psychopharmacology 1999; 146: 24-32.

46 Saslow MG. Effects of components of displacement-step stimuli upon latency of saccadic eye movements. Journal of the Optical Society of America 1967; 57: 10241029.

47 Fischer B, Weber H. Express saccades and visual attention. Behavioral and Brain Sciences 1993; 16: 553-610.

48 Faria Jr. AJP, Machado-Pinheiro W. Looking for the gap effect in manual responses and the role of contextual influences in reaction time experiments. Brazilian Journal of Medical and Biological Research 2004; 37: 1175-1184. 
Canto-Pereira et al. Alcohol and attention - Human \& Experimental Toxicology

49 Mayfrank L, Mobashery M, Kimming H, Fischer B. The role of fixation and visual attention in the occurrence of express saccades in man. European Archives of Psychiatry and Neurological Science 1986; 235: 269-275.

50 Mackeben M, Nakayama K. Express attentional shifts. Vision Research 1993; 33(1): 85-90.

51 Canto-Pereira LHM, Ranvaud R. Splitting the beam? Evidence of divided visual attention assessed through a geostatistical method. Perception 2005; 34: 178-179. 
Canto-Pereira et al. Alcohol and attention - Human \& Experimental Toxicology

\section{Legends}

\section{Figure 1.}

A: grid of positions where stimuli were presented in the experiments I. The central area of the computer screen, where participants must direct their attention, were densely distributed when compared to the sparsely points in the rest of the screen; $\mathrm{x}$ and $\mathrm{y}$ in pixels units.

B: experiment's II positions grid where stimuli were presented. Here there are two regions densely distributed, where participants must direct their attention, both right and left and the same sparsely distributed points on the rest of the screen as in experiment $\mathrm{I}$; $\mathrm{x}$ and $\mathrm{y}$ in pixels units.

Figure 2: Data (mean RTs in ms) from Experiments I and II for controls and alcohol group.

\section{Figure 3.}

A: Mean RTs for ROIs in Experiments I and II for the control group.

B: Mean RTs for ROIs in Experiments I and II for the alcohol group.

\section{Figure 4.}

A: pixel map of experiment I for controls shows clearly shorter RTs in the center coincident with the attentional focus;

B: experiment I for alcohol group shows the same pattern but with a slightly bigger focus;

C: in experiment II controls were able to orient their attention to the two lateral targets leaving the central region unattended;

D: experiment's II pixel map shows clearly a remarkable difference when compared to controls, it was not possible to participants disengage their attentional focus from the center where it was located the fixation point, i.e. where their gaze were located. 
Canto-Pereira et al. Alcohol and attention - Human \& Experimental Toxicology

Figures

Figure 1

A

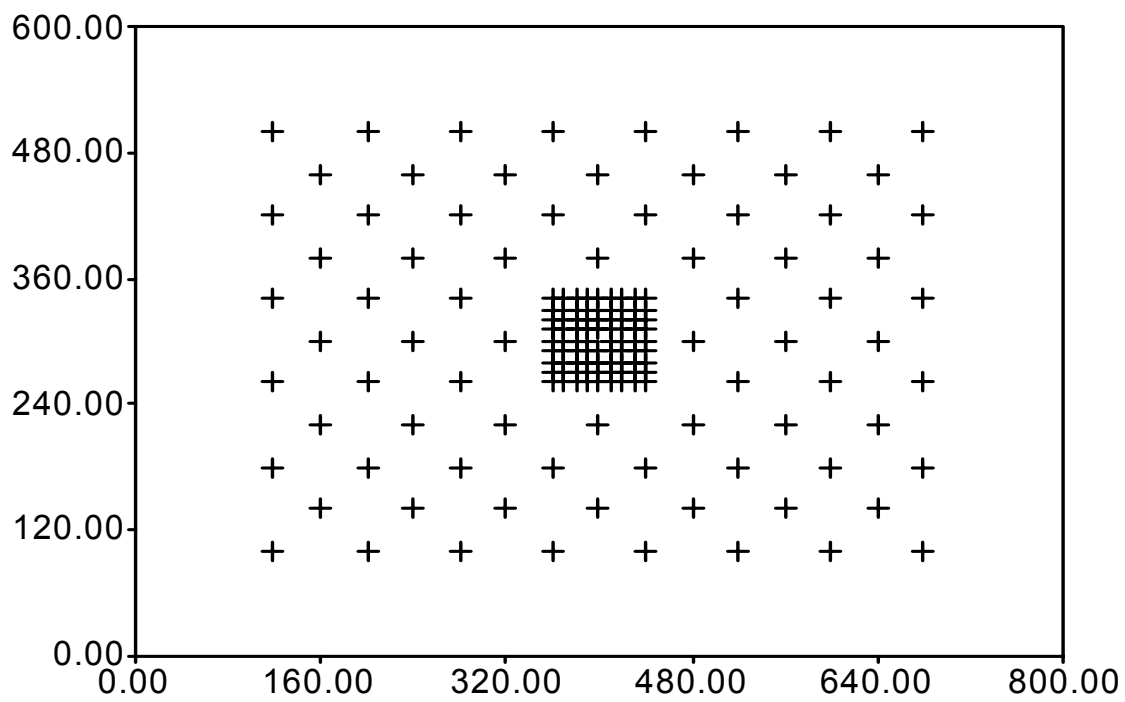

B

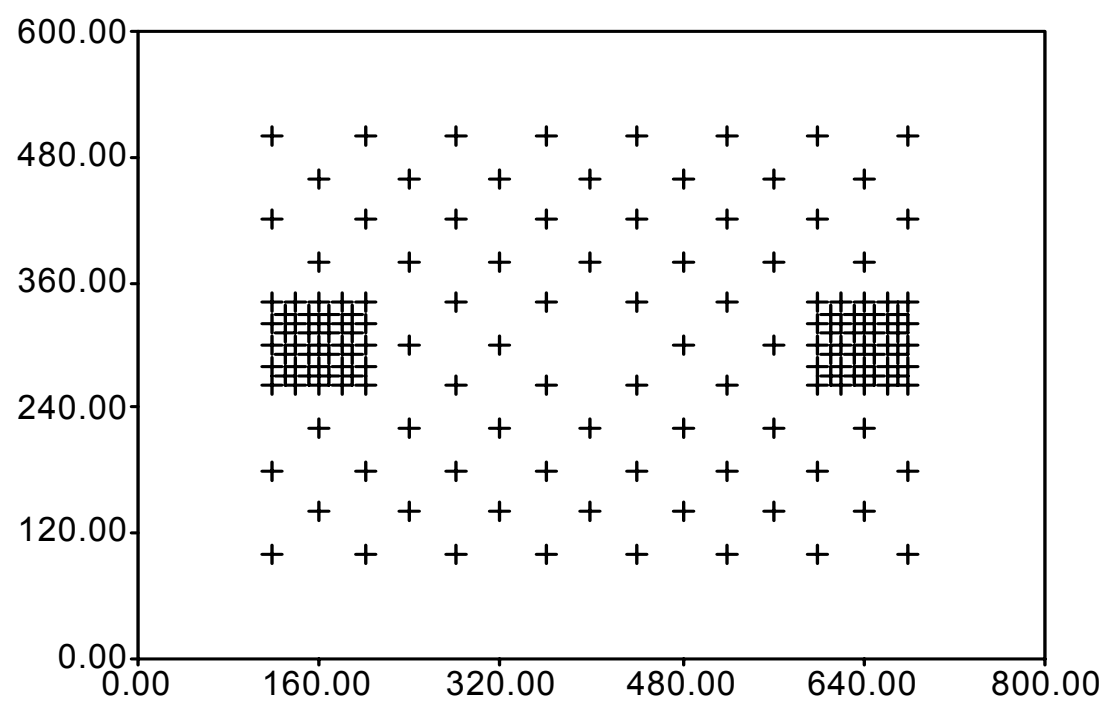


Figure 2

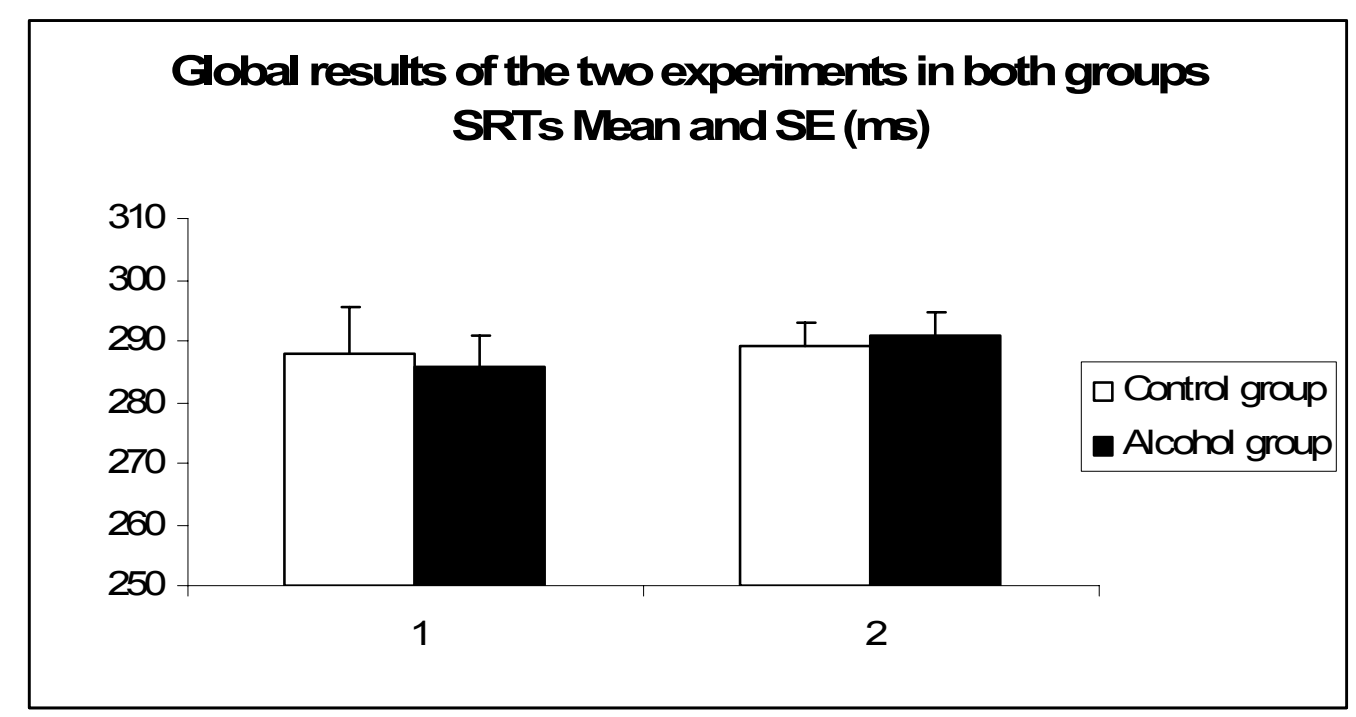


Canto-Pereira et al. Alcohol and attention - Human \& Experimental Toxicology

Figure 3.

A.

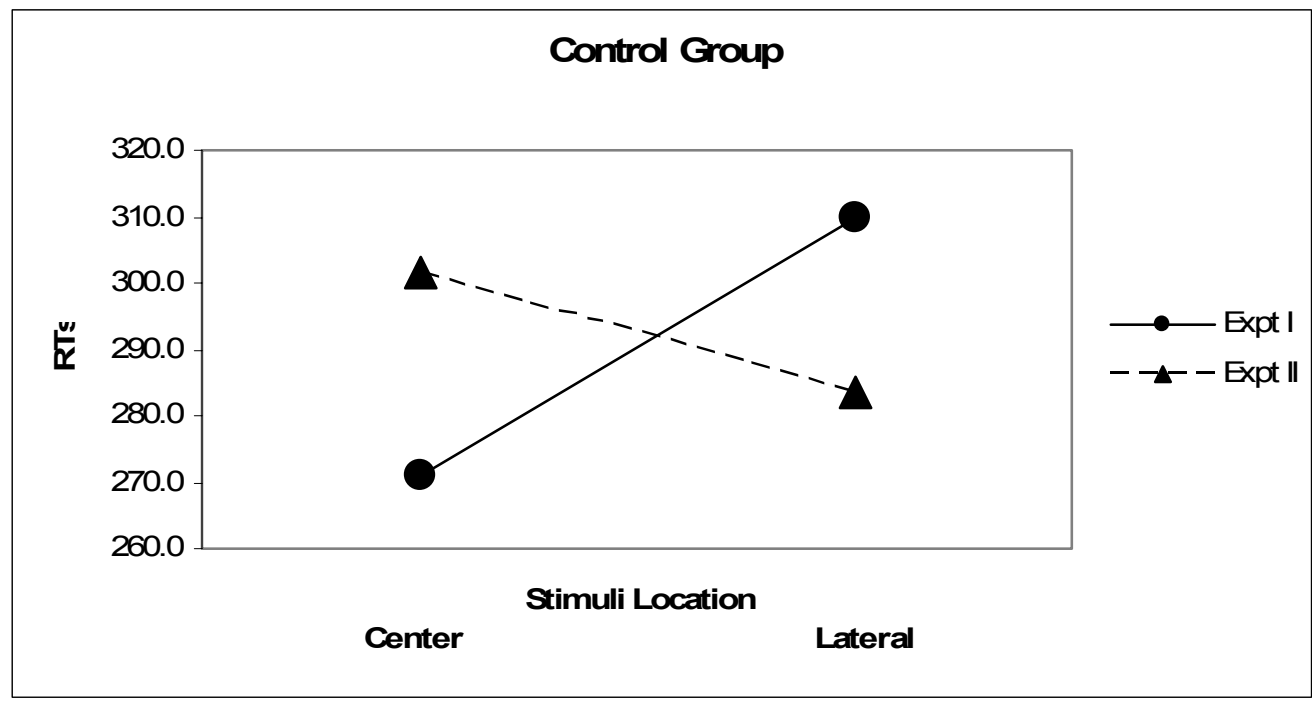

B.

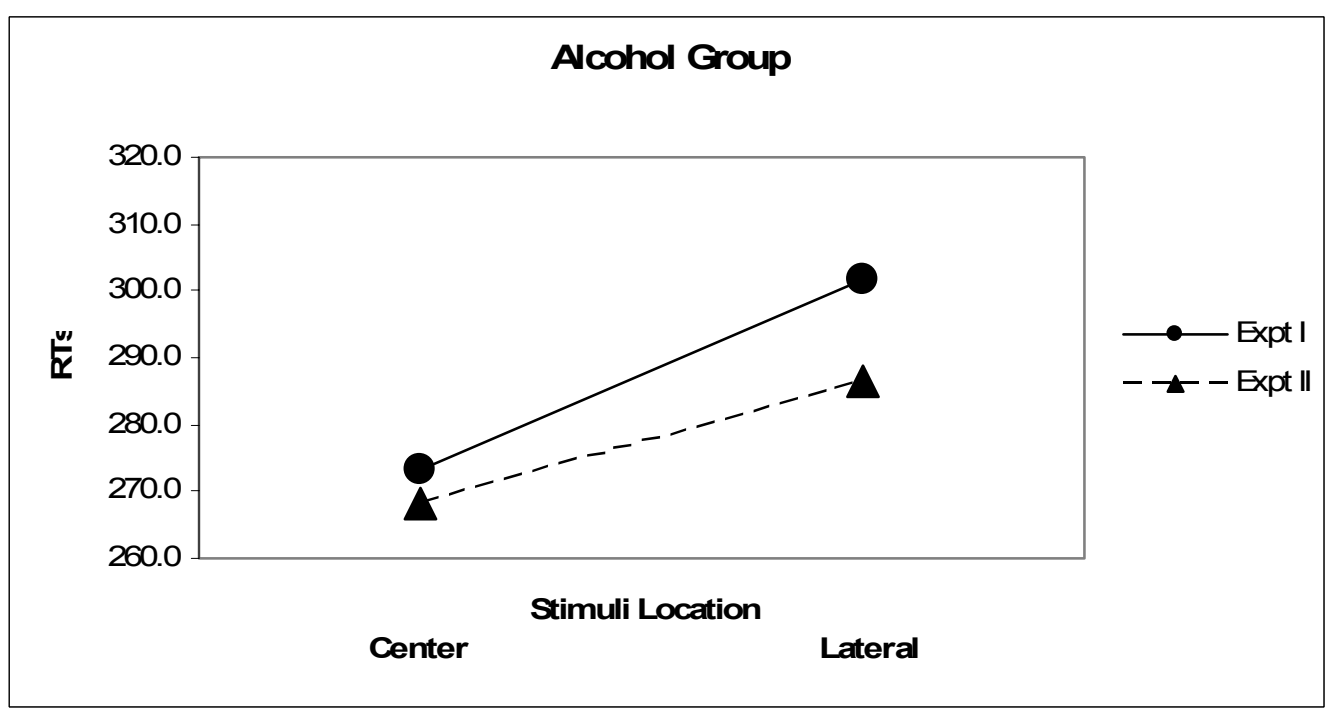


Figure 4.

A
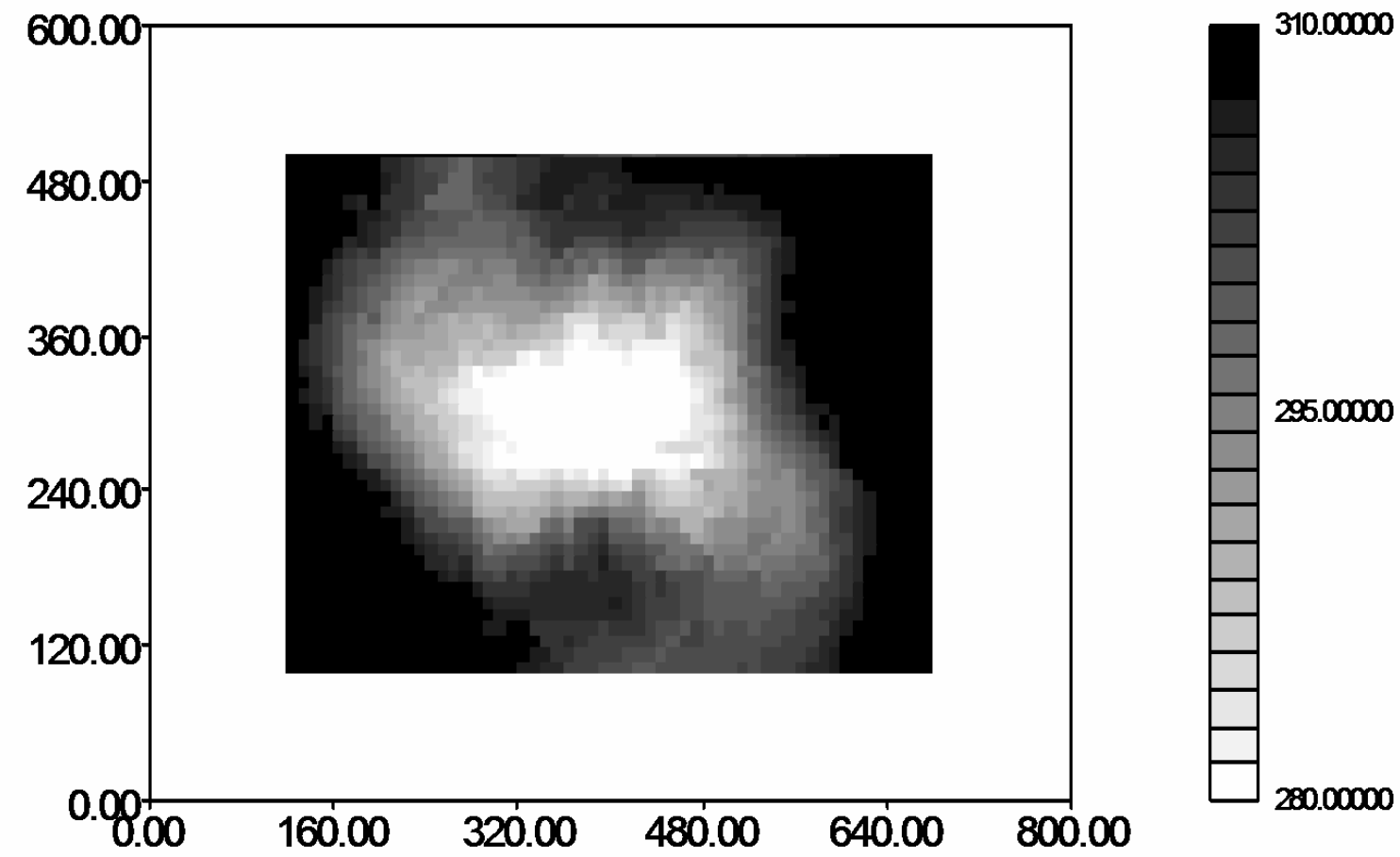

B
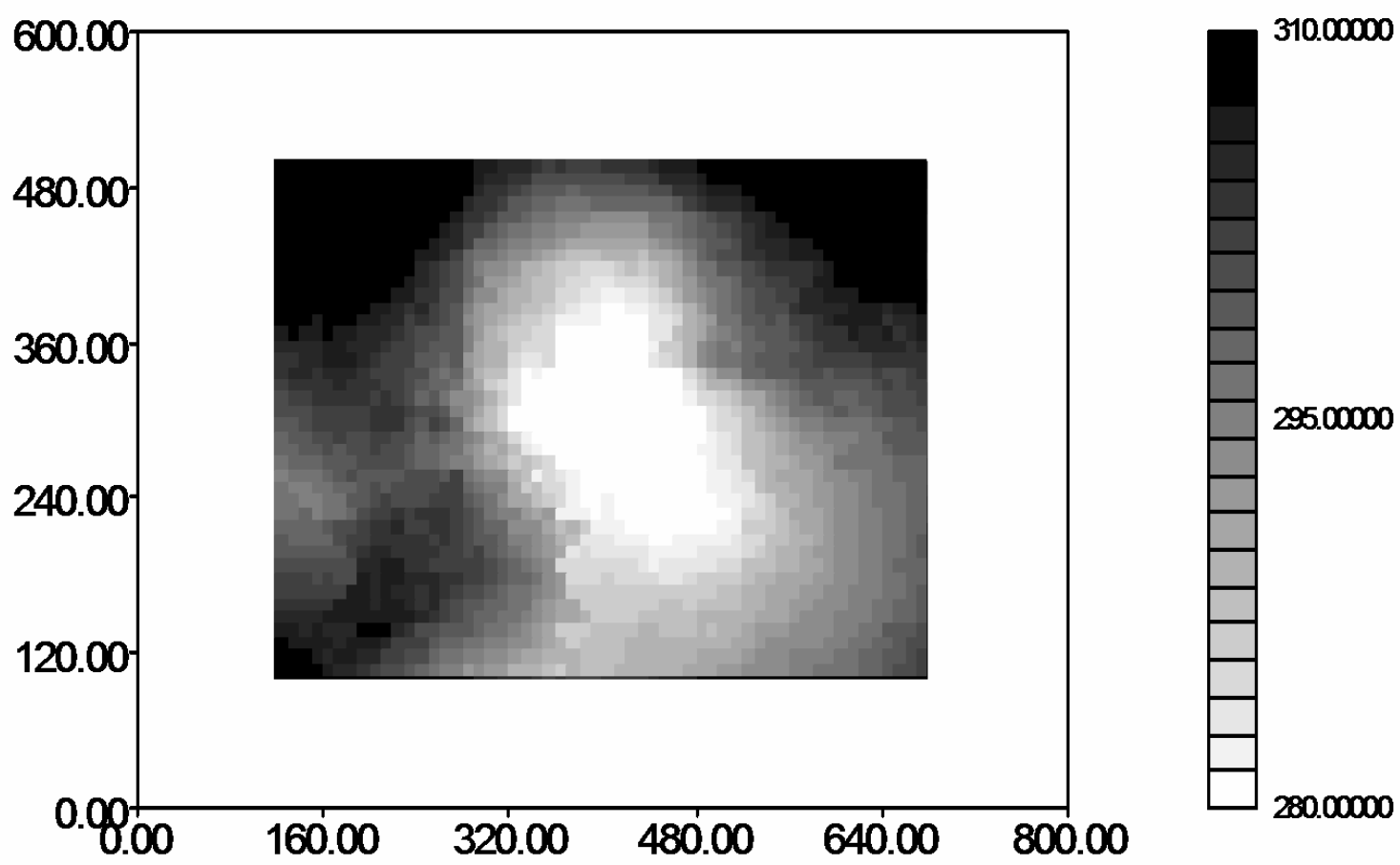
C
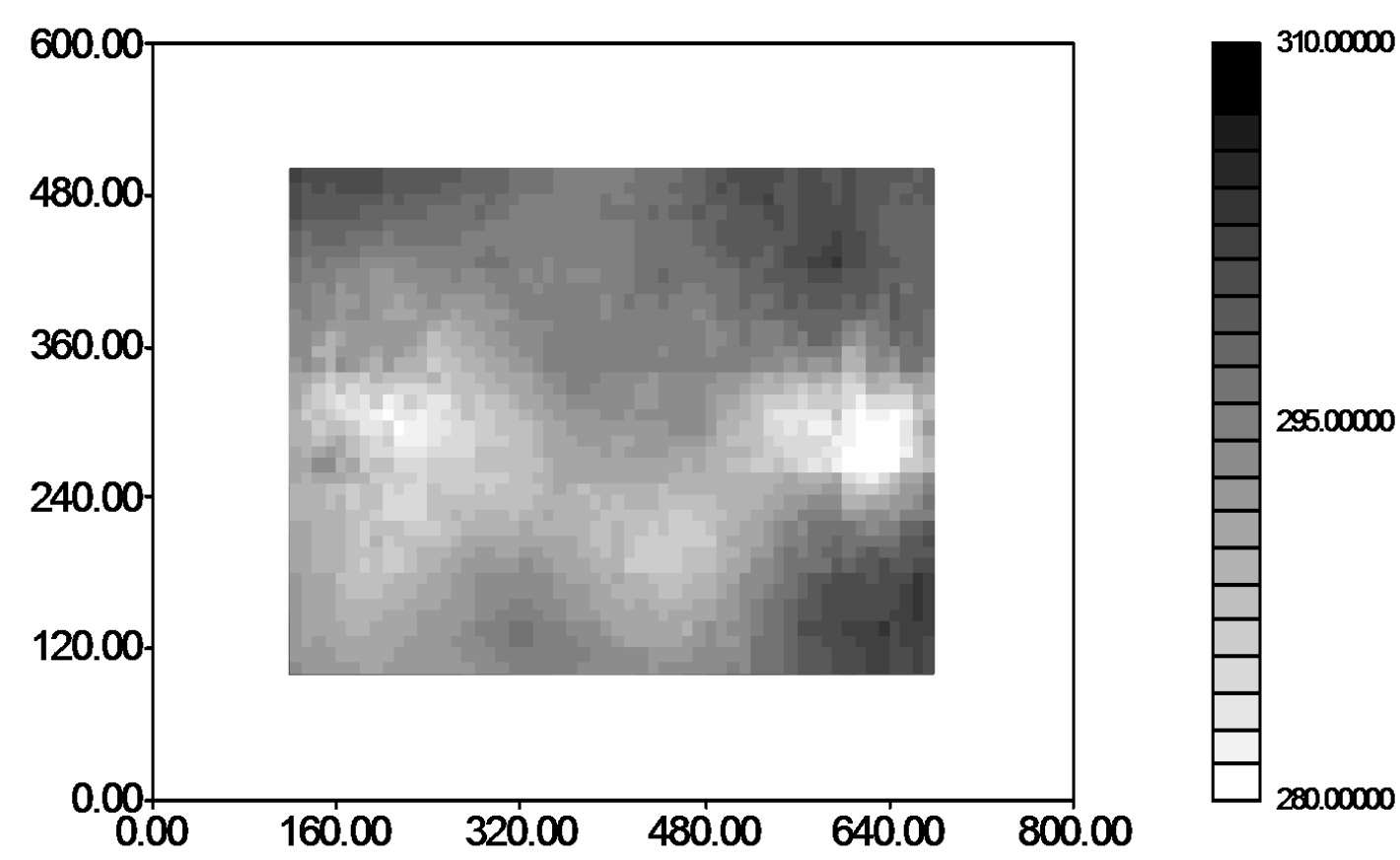

$\mathrm{D}$
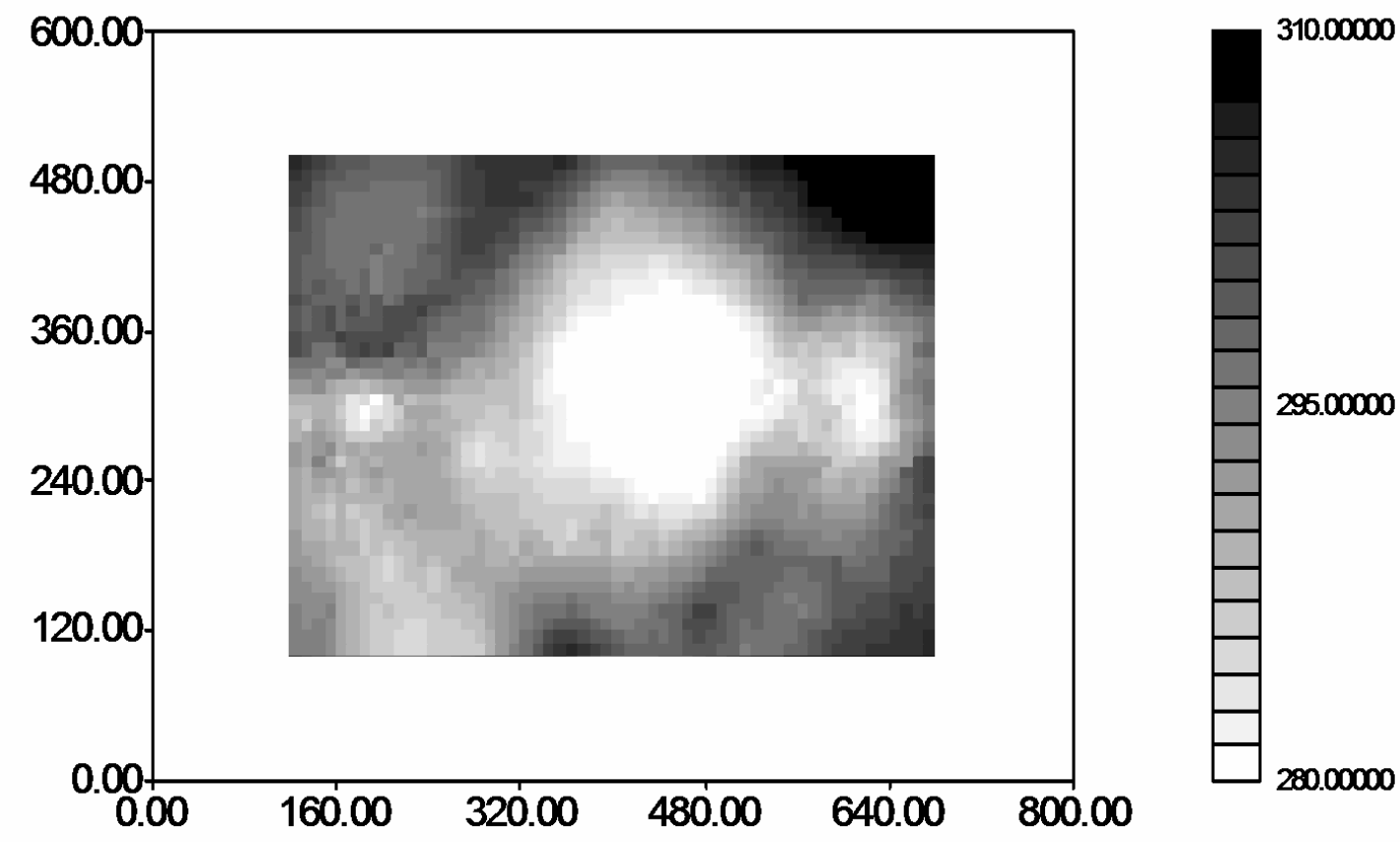